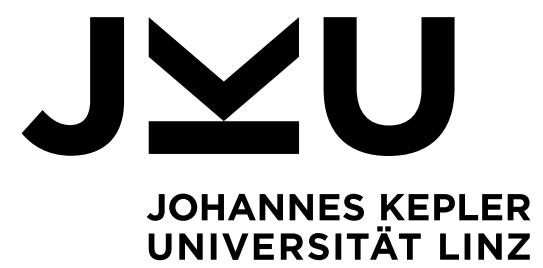

Eingereicht von

Rafael Wiedmer Chaparro, BSc

Angefertigt am

Ordensklinikum Linz

Beurteiler / Beurteilerin

Prim. Univ. Prof. Dr. R. Schöfl

\title{
RETROSPEKTIVE ANALYSE DER PERORALEN ENDOSKOPISCHEN MYOTOMIE (POEM) ZUR THERAPIE DER ACHALASIE AM ORDENSKLINIKUM LINZ
}

Mitbetreuung

Dr. T. Fritz, PhD

Mai 2020

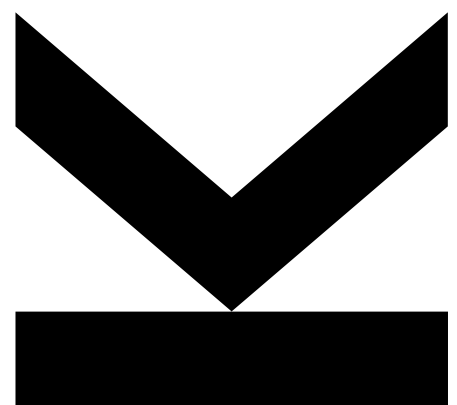

Masterarbeit

zur Erlangung des akademischen Grades

Dr. med. univ.

im Masterstudium

Humanmedizin 


\section{EIDESSTATTLICHE ERKLÄRUNG}

Ich erkläre an Eides statt, dass ich die vorliegende Masterarbeit selbstständig und ohne fremde Hilfe verfasst, andere als die angegebenen Quellen und Hilfsmittel nicht benutzt bzw. die wörtlich oder sinngemäß entnommenen Stellen als solche kenntlich gemacht habe.

Die vorliegende Masterarbeit ist mit dem elektronisch übermittelten Textdokument identisch.

Ort, Datum

Unterschrift 


\section{Inhaltsverzeichnis}

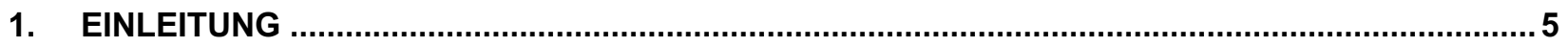

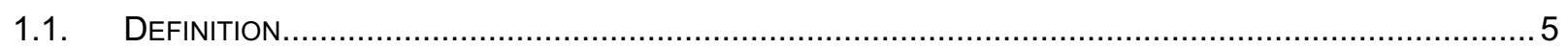

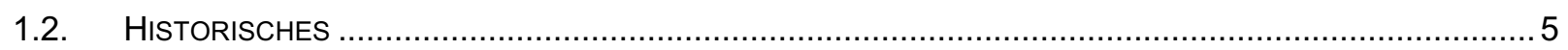

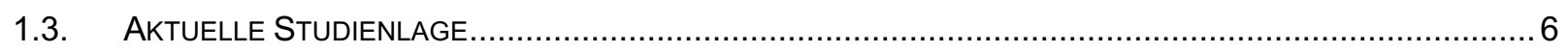

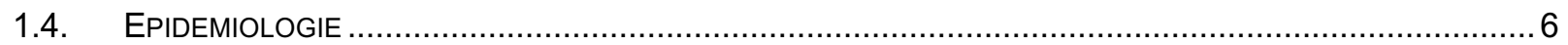

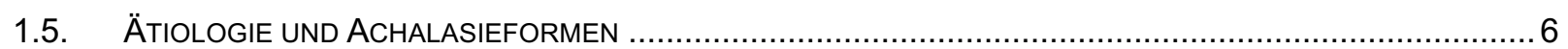

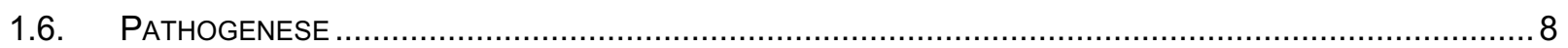

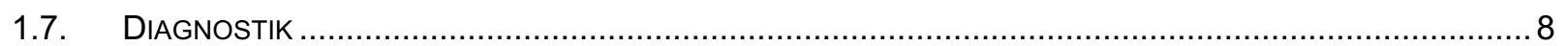

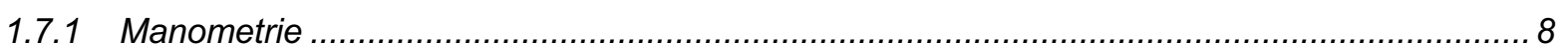

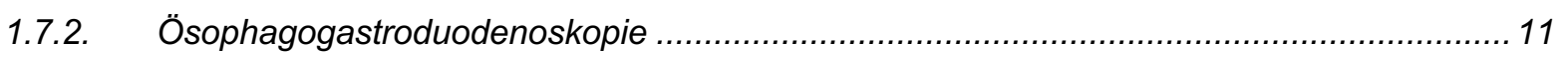

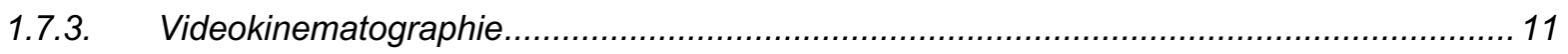

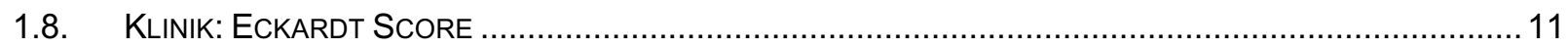

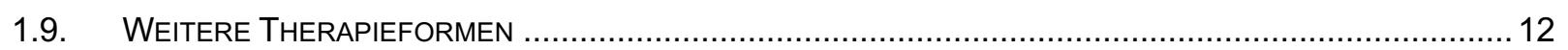

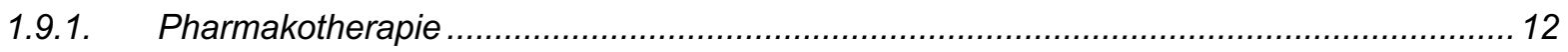

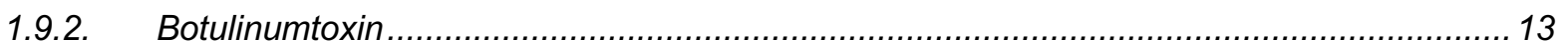

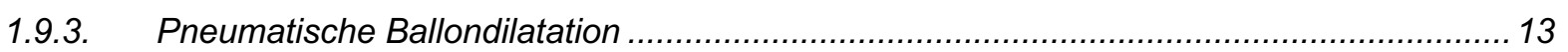

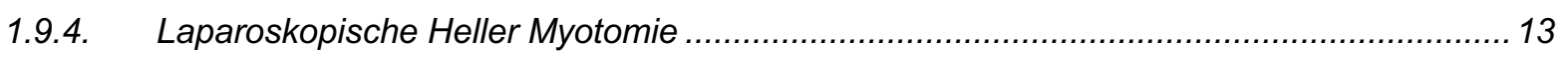

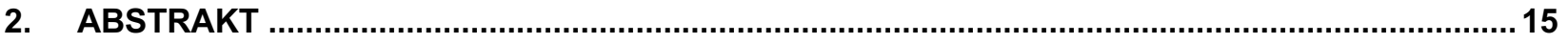

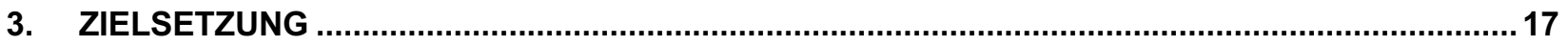

4. MATERIAL UND METHODIK

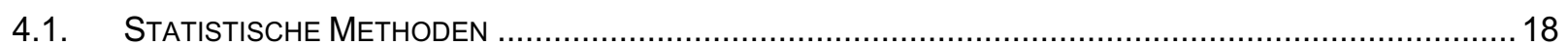

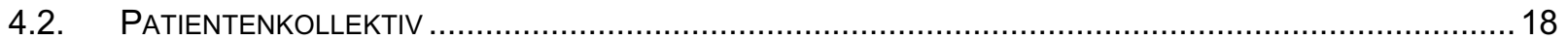

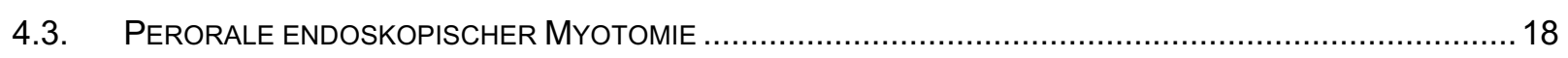

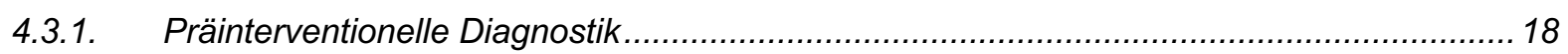

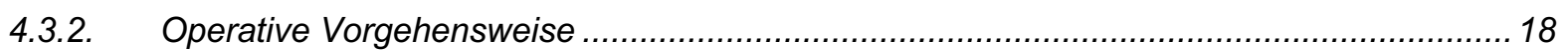

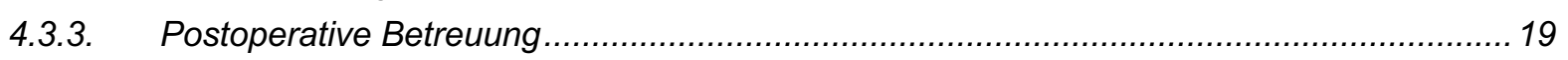

5. ERGEBNISSE

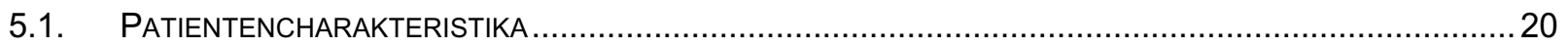

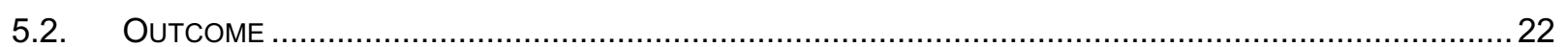

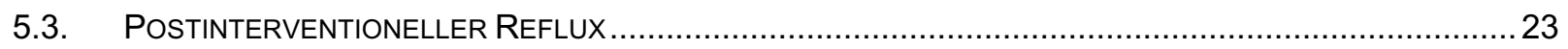

5.3.1. Postinterventionelle Refluxkrankheit bezogen auf das Patientenalter ................................ 25

5.3.2. Postinterventionelle Refluxkrankheit in Zusammenhang mit dem präinterventionellen

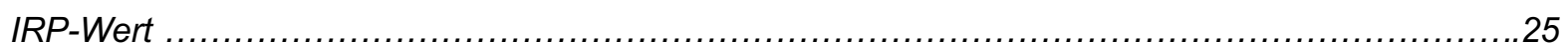

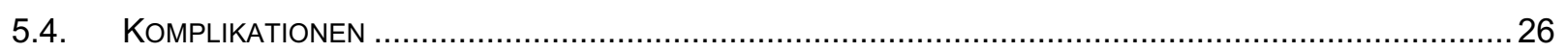

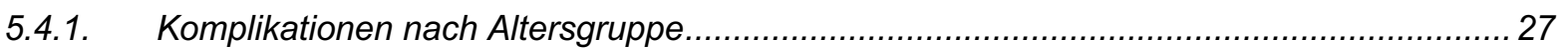

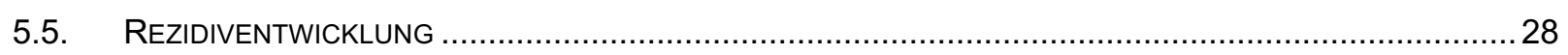

5.5.1. Rezidiventwicklung bezogen auf das Patientenalter .................................................... 29

5.5.2. Rezidiventwicklung in Zusammenhang mit präinterventionellen klinischen Beschwerden 29

5.6. LANGZEITOUTCOME DER POEM AM ORDENSKLINIKUM LINZ .................................................. 30

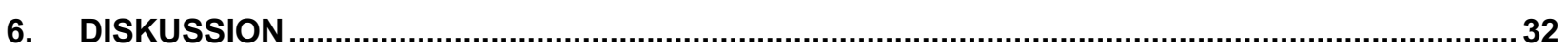

6.1. VoR- Und NACHTEILE der POEM GegenÜBer ANDEREN Methoden.............................................. 33

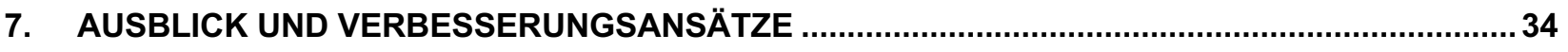




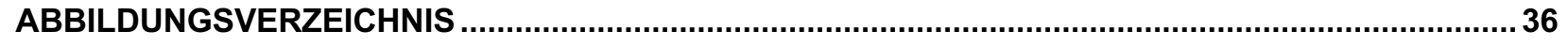

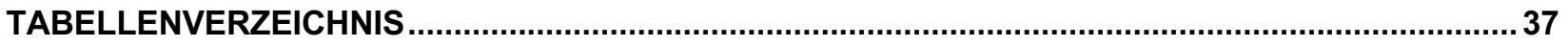

ABKÜRZUNGSVERZEICHNIS

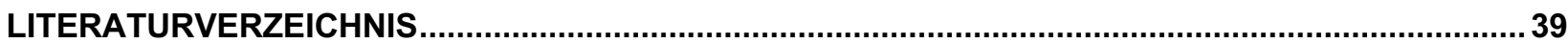




\section{Einleitung}

\subsection{Definition}

Die Achalasie bezeichnet eine Motilitätsstörung des Ösophagus, welche durch eine gestörte Relaxationsfähigkeit und erhöhten Tonus des unteren Ösophagussphinkters (UÖS) sowie mangelnde Peristaltik gekennzeichnet ist. Pathophysiologisch kommt es zu einem Verlust von inhibitorischen Ganglien im Plexus Myentericus des Ösophagus. Leitsymptom der Erkrankung ist die Dysphagie. ${ }^{1}$

Neben den schon länger bekannten therapeutischen Methoden, wie der laparoskopischen Heller Myotomie (LHM) und der endoskopischen Ballondilatation (EBD), steht seit einigen Jahren die perorale endoskopische Myotomie (POEM) zur Verfügung. Die POEM zeichnet sich vor allem durch ihren minimalinvasiven Ansatz aus. In der Theorie vereint sie die Minimalinvasivität der EBD mit dem durch die Myotomie bedingten längerfristigen Therapieerfolg der LHM.

Der erste Schritt bei der Durchführung einer POEM ist eine Inzision in die Mukosa des Ösophagus, anschließend präpariert man bis in die Submukosa. In den entstehenden Spalt wird das Endoskop eingeführt. Von dort aus wird eine fortlaufende Dissektion der Submukosa durchgeführt. Dabei bahnt sich der Endoskopist einen Tunnel unter der Mukosa, an der Muskularis propria entlang, in Richtung Magen. Zum Schneiden wird hochfrequenter Strom verwendet. ${ }^{2}$

Der Tunnel wird wenige Zentimeter über den ösophagogastralen Übergang hinaus gebildet. Anschließend erfolgt eine Myotomie der Ringmuskulatur unter Erhalt der Längsmuskulatur des UÖS. In den meisten Fällen wird die Myotomie auf einige Zentimeter proximal und distal des Sphinkters ausgedehnt. ${ }^{3}$

\subsection{Historisches}

Das Jahr 2008 markiert das Debüt der POEM. Die ersten Eingriffe werden von H. Inoue et al. in Japan in einer Fallreihe mit 17 Patienten dokumentiert. In allen Fällen kann der Eckardt Score (ES) sowie der Muskeltonus des UÖS signifikant gesenkt werden. Bei keinem Eingriff treten Komplikationen auf. Lediglich bei einer Patientin tritt fünf Monate postinterventionell eine Refluxösophagitis auf, die mittels Protonenpumpen-Inhibitoren gut behandelbar ist. ${ }^{4}$

Im europäischen Raum lieferten 2012 von Renteln et. al. erste Ergebnisse. Bei 16 mittels POEM therapierten Patienten wurde in $94 \%$ der Fälle ein Therapieerfolg erzielt, definiert als ES $\leq 3$ drei Monate postinterventionell. Kein Patient entwickelte eine Refluxösophagitis. Bei einem Patienten findet sich eine erosive Läsion des Ösophagus in einer Follow-Up Ösophagogastroduodenoskopie. ${ }^{5}$

In beiden Fallreihen erreicht die POEM ähnliche Erfolgsraten wie die LHM, zumindest was die Kurzzeitergebnisse angeht. Aussagen über das Langzeit-Outcome können zu diesem Zeitpunkt nicht getroffen werden. 


\subsection{Aktuelle Studienlage}

Eine 2019 publizierte, multizentrische, retrospektive Studie von Shiwaku und Inoue et al. analysiert Daten von peroralen endoskopischen Myotomien, die zwischen September 2008 und Oktober 2015 an acht japanischen Zentren durchgeführt wurden. Die Studie beinhaltet 1346 Achalasie Patienten mit einem durchschnittlichem Alter von 47 Jahren. Die Frauen hielten einen Anteil von 54\%, die Männer entsprechend $46 \%$ an der Gesamtheit der Studienteilnehmer. Einige Probanden waren bereits vortherapiert (28\% erhielten bereits eine Ballondilatation, 3\% eine laparoskopische Heller Myotomie). Die Therapie mittels POEM war 3 Monate postoperativ in 95,1\% der Fälle und ein Jahr postoperativ in $94,7 \%$ der Fälle erfolgreich (definiert als Eckardt-Score $\leq 3$ ). In 3,7\% der Fälle traten Komplikationen auf, die jedoch alle konservativ behandelt werden konnten. Innerhalb von sechs Monaten nach POEM erhielten 1176 der Studienteilnehmer eine Kontroll-Endoskopie. 63\% der Patienten zeigten eine erosive Refluxkrankheit (erosive reflux disease, ERD) im Sinne der Los Angeles Klassifikation (33\% Grad A; 24\% Grad B; 6\% Grad C; 0,2\% Grad D). 14,8\% der Patienten präsentierten eine symptomatische gastroösophageale Refluxkrankheit (gastroesophageal reflux disease, GERD). Patienten mit erosiver Refluxkrankheit sowie Patienten mit symptomatischer gastroösophagealer Refluxkrankheit wurden erfolgreich mit Protonenpumpen-Inhibitoren behandelt. Die Studie bestätigt die Effizienz und Sicherheit der Methode. Aufgrund der Ergebnisse dieser groß angelegten Studie unterstützen die japanischen Autoren die POEM als Erstlinientherapie für die Achalasie. ${ }^{6}$

\subsection{Epidemiologie}

Die Achalasie ist mit einer Inzidenz von ca. 1/100'000 pro Jahr bei Erwachsenen und ca. 0,2/100'000 pro Jahr bei Kindern unter 16 Jahren eine eher seltene Erkrankung. Frauen sind geringfügig häufiger betroffen als Männer. ${ }^{7}$ Alle Ethnien sind gleich betroffen, jedoch steigt die Inzidenz mit dem Alter. Das durchschnittliche Alter zum Zeitpunkt der Diagnose liegt bei über 50 Jahren. Bei Patienten über 80 Jahre ist die Anzahl an Neuerkrankungen pro Jahr im Vergleich zum Durchschnitts-Erwachsenen um das 17-fache höher, also bei $17 / 100{ }^{\prime} 000 .^{8}$ Es finden sich zwei Altersgipfel, einen bei $60-80$ Jahren und einen zweiten bei 30-40 Jahren. Die Prävalenz beläuft sich auf ungefähr 10/100'000, Tendenz leicht steigend..$^{8,9,10}$

\section{5. Ätiologie und Achalasieformen}

Die Achalasie kann nach ihrer Entstehungsursache in eine primäre, diese wird meist als idiopathische Form bezeichnet, und in eine sekundäre Form, die sog. Pseudoachalasie, eingeteilt werden. Es handelt sich um eine entzündliche Erkrankung, die mit progressiver Degeneration von Neuronen im Plexus Myentericus „Auerbach“ der Ösophaguswand einhergeht. Dies führt zu einem Spasmus des UÖS und einem Verlust der Peristaltik im Bereich des distalen Ösophagus. ${ }^{11}$

Bei der idiopathischen Achalasie ist die Ursache für die Entzündungsreaktion unbekannt. Einige Hinweise für die Entstehung gibt es allerdings.

Eine unter Experten mehrheitlich akzeptierte These ist die der autoimmunen Genese. 
So wurde bei einer mexikanischen Studie von Furuzawa-Carballeda et al. ${ }^{11}$ ein prozentualer Anstieg von zwei CD4+ T-Zell-Subpopulationen bei Achalasie Patienten beobachtet. Dabei handelt es sich um TH17 und TH22, welche u.a. IL-17A und IL-22 sezernieren. Beide sind bereits mit diversen Autoimmunerkrankungen wie dem Sjögren-Syndrom, Multipler Sklerose oder rheumatoider Arthritis assoziiert. In Biopsien des UÖS waren IL-17A- und IL-22produzierende Zellen der zelluläre Hauptanteil der entzündlichen Infiltrate. In der gleichen Studie wurde ein signifikanter Anstieg der Autoantikörper gegen den Plexus Myentericus mittels eines Immunoblot-Verfahrens nachgewiesen.

Ein Fallbericht aus Kuwait verdeutlicht die autoimmune Genese. Eine Patientin entwickelt im Alter von 16 Jahren eine chronisch rezidivierende Achalasie. Zehn Jahre später manifestiert sich bei ihr eine schwere autoimmune Hämophilie A, welche mit einer Hochdosis-Steroidtherapie über sieben Monate behandelt wird. Seit der siebenmonatigen immunsuppressiven Therapie und zwei weiteren Jahren nach ihrer Beendigung sind sowohl die Hämophilie $A$ als auch die Achalasie in Remission. ${ }^{12}$ Ein Ansprechen auf eine immunsuppressive Therapie würde für eine autoimmune Genese sprechen.

Zudem ist bekannt, dass Patienten mit Autoimmunerkrankungen ein erhöhtes Risiko besitzen weitere Autoimmunerkrankungen zu entwickeln, was in beiden Studien gehäuft der Fall war. In der mexikanischen Studie litten vier von neunzehn zufällig ausgewählten Achalasie Patienten zusätzlich an einer Autoimmunerkrankung. ${ }^{11}$ Dieses gehäufte Auftreten von Autoimmunerkrankungen unterstützt die Theorie der autoimmunen Genese.

Die stark erhöhte Prävalenz von HLA-DQ-Varianten ${ }^{13}$ zusammen mit erhöhter Autoantikörper-Prävalenz spricht für eine autoimmune Komponente.

Der Trigger dafür ist womöglich eine Virusinfektion. Von 14 gewonnenen Biopsien aus dem UÖS wurde bei $100 \%$ eine HSV-1-Infektion mittels PCR, RT-PCR und Immunhistochemie nachgewiesen. In einem anderen Versuchsaufbau wurden die orale und ösophageale Mukosa als bevorzugte Eintrittspforten des Virus aufgezeigt. Bekannt ist außerdem, dass Herpes-Viren einen starken Tropismus für Nervenfasern besitzen, in deren Zellkernen das Virus in latenter Form verbleibt. ${ }^{11}$

Die Auslöser der sekundären Achalasie sind vielfältig und beinhalten alle Erkrankungen, die, verglichen mit der primären Form, identische oder sehr ähnliche Motilitätsstörungen des Ösophagus verursachen.

In einer Studie wurden 14 Jahre lang Patienten mit Verdacht auf Achalasie beobachtet. Von letztendlich 167 in die Studie aufgenommenen Patienten stellte sich bei nur sechs Patienten eine sekundäre Form dar, was bereits Aufschluss über die Häufigkeitsverteilung der beiden Formen gibt. ${ }^{14}$ Die sekundäre Form kommt mit den Zahlen aus dieser Studie auf eine statistische Inzidenz von unter 1/1'000'000 und ist somit eine Rarität. Ihre mit Abstand häufigste Ursache ist eine maligne Erkrankung. Bei allen sechs Patienten der Studie mit einer sekundären Achalasieform war die Ursache eine Tumorerkrankung. Davon war bei fünf Patienten der Auslöser ein Adenokarzinom des Magenfundus und bei einem Patienten fand sich ein Plattenepithelkarzinom des Ösophagus. Das Magenkarzinom scheint somit die häufigste Ursache einer Pseudoachalasie zu sein. ${ }^{14}$

Die Tumore selbst oder befallene Lymphknoten im unteren Mediastinum drücken im weiteren Verlauf auf Nerven im Bereich der Kardia, was zu einem ähnlichen Krankheitsbild wie bei einer idiopathischen Form führt. 
Weitere bewiesene Auslöser für eine sekundäre Form sind unter anderem Sarkoidose, Amyloidose, Neurofibromatose, Multiple Endokrine Neoplasie, Lungenkarzinom, chronisch intestinale Pseudoobstruktion und Morbus Fabry. ${ }^{15-21}$

\subsection{Pathogenese}

Die Pathogenese der Achalasie ist derzeit noch nicht vollständig geklärt, jedoch existieren nachvollziehbare Theorien.

Als Trigger der idiopathischen Form könnte eine Virusinfektion (z.B. HSV-1) fungieren. Liegt nun zusätzlich zur HSV-1-Infektion eine genetische Prädisposition in Form einer HLA-DQVariante vor, kommt es an dieser Stelle womöglich zu einem Verlust der Immuntoleranz und zur Ausbildung von inflammatorischen Infiltraten in der Ösophaguswand, welche eine bevorzugte Eintrittspforte für das Virus darstellt. ${ }^{11}$

Die Entzündungsreaktion führt zu einer Degeneration der Neurone im Plexus Myentericus. Besonders betroffen scheinen die Stickstoffmonoxid-produzierenden inhibitorischen Neurone zu sein. Eben jene NO-Produktion ist verantwortlich für eine Relaxation der glatten Muskulatur im UÖS. Cholinerge Neurone, die für die Kontraktion der Sphinktermuskulatur zuständig sind, bleiben im Verhältnis dazu eher verschont. ${ }^{22}$ Daraus folgt ein Ungleichgewicht aus inhibierenden und induzierenden Neuronen, was letztlich zum Verlust der Relaxationsfähigkeit bzw. zum Spasmus des unteren Sphinkters führt. In der glatten Muskulatur des Ösophaguskörpers resultiert dies in einer Aperistaltik. Im fortgeschrittenen Stadium kann sich eine enorme Ösophagusdilatation, sog. Megaösophagus, darstellen, zusammen mit einer Sigmoid-Verformung der Speiseröhre. Die genaue Ausprägung ist abhängig vom Ausmaß und von der Lokalisation der Neurodegeneration. ${ }^{23}$

\subsection{Diagnostik}

\subsubsection{Manometrie}

Die Manometrie gilt als die sensitivste Methode und als Gold-Standard der Achalasie-Diagnostik. Hierbei wird dem Patienten eine Sonde nasal in den Ösophagus eingeführt und zum Mageneingang vorgeschoben. Dann wird der Patient aufgefordert wiederholt in kleinen Abständen zu schlucken. Die Sonde besitzt mehrere Sensoren, die die Druckunterschiede während des Schluckakts messen, welche dann an einem Computer graphisch dargestellt werden.

Zu den auschlaggebenden Merkmalen bei der Befundung einer Achalasie gehört eine Aperistaltik der distalen zwei Drittel des Ösophagus. Dabei kann der Schluckakt keine Reaktion der glatten Muskulatur nach sich ziehen oder aber es folgt eine zeitgleiche Kontraktion des gesamten Ösophagus.

Als zentrales Kriterium, das bei der Unterscheidung von Differentialdiagnosen eine wichtige Rolle spielt, gilt die unvollständige Relaxation des UÖS. Beim Gesunden kommt es nach dem Schluckakt zu einer Entspannung des Schließmuskels, was mit einem Druck von $<8 m m H g$ über Magendruck einhergeht. Bei Achalasie Patienten liegt der niedrigste gemessene Wert der Entspannung nach dem Schluckakt bei mind. $>8 \mathrm{mmHg}$ über Magendruck. 
Häufig wird zudem ein erhöhter Tonus des UÖS in Ruhe beobachtet, was jedoch kein notwendiges Kriterium für eine Diagnose ist. ${ }^{24}$

In der modernen High-Resolution-Manometrie (HRM) ist neben mehr eingebauten Drucksensoren, und damit einer höheren Auflösung, auch die Beobachtungen weiterer Parameter möglich. So erlaubt die HRM die Einteilung der Achalasie in die drei Subtypen der international anerkannten Chicago-Klassifikation.

Bei der HRM kann der sog. Integrierte Relaxationsdruck (IRP) ermittelt werden. Dieser ist ein Index für Aperistaltik und gestörte Relaxation im gastroösophagealen Übergang. Eine Erhöhung des mittleren IRP ist typisch für Achalasie. Ein weiterer Parameter in der HRM ist das sog. Distale Kontraktionsintegral $(\mathrm{DCl})$. Der $\mathrm{DCl}$ ist ein Marker für die Intensität der distalen Ösophaguskontraktion und spielt eine Rolle bei der Einteilung in die jeweiligen Achalasiesubtypen. ${ }^{25}$

Am Ordensklinikum Linz ist die HR-Manometrie als internationaler Gold-Standard die Methode der Wahl zur Diagnose der Achalasie und dient zur Indikationsstellung der Therapie.

\subsubsection{Achalasie Typ 1}

Typ I wird oft auch als „klassische“ Achalasie bezeichnet.

Hierbei führt das Schlucken zu keiner nennenswerten Veränderung des Ösophagustonus. Es kommt zu einem vollständigen Peristaltikverlust mit einem $\mathrm{DCl}<100 \mathrm{mmHg}^{25}$

Folgende Darstellung des Uniklinikums Freiburg dient zur Veranschaulichung. ${ }^{26}$

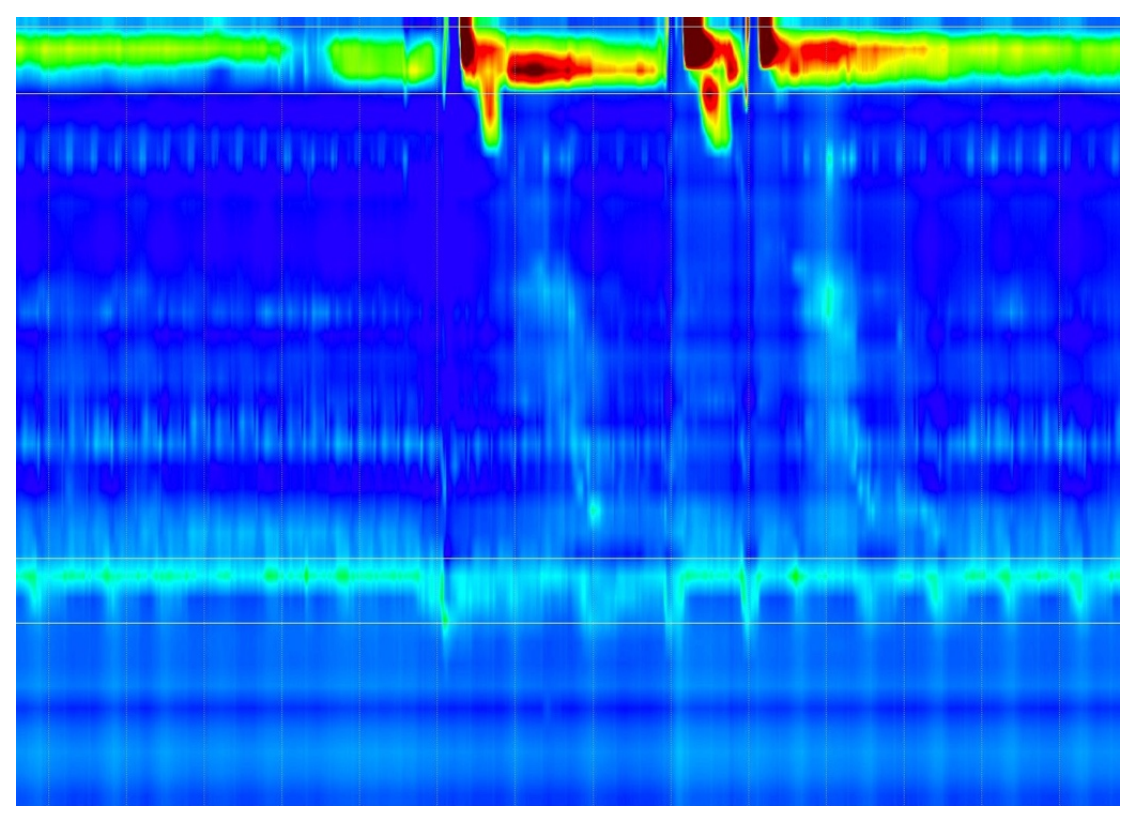

Abb. 1: Achalasie Typ 1 (Quelle: Uniklinik Freiburg) 


\subsubsection{Achalasie Typ 2}

Das Schlucken resultiert in einer pan-ösophagealen Kontraktion bzw. einer simultanen Druckerhöhung des gesamten Ösophagus, was zur Aperistaltik führt. ${ }^{25}$

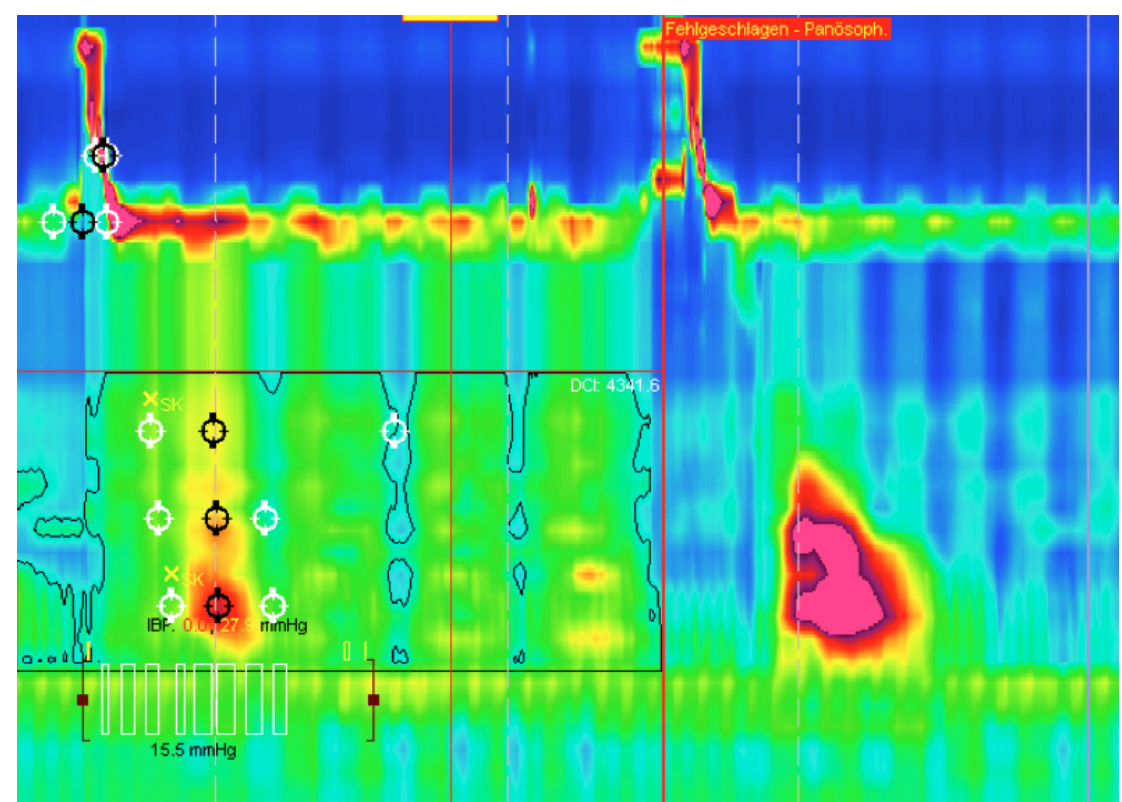

Abb. 2: Achalasie Typ 2 (Quelle: Ordensklinikum Linz)

\subsubsection{Achalasie Typ 3}

Dieser Subtyp wird oft auch „spastische“ Achalasie genannt. Das Schlucken ist gefolgt von Lumen-verschließenden Spasmen. Die Peristaltik ist gestört und die spastischen Kontraktionen weisen einen $\mathrm{DCl}$ von $>450 \mathrm{mmHg}_{\text {auf. }}{ }^{25}$

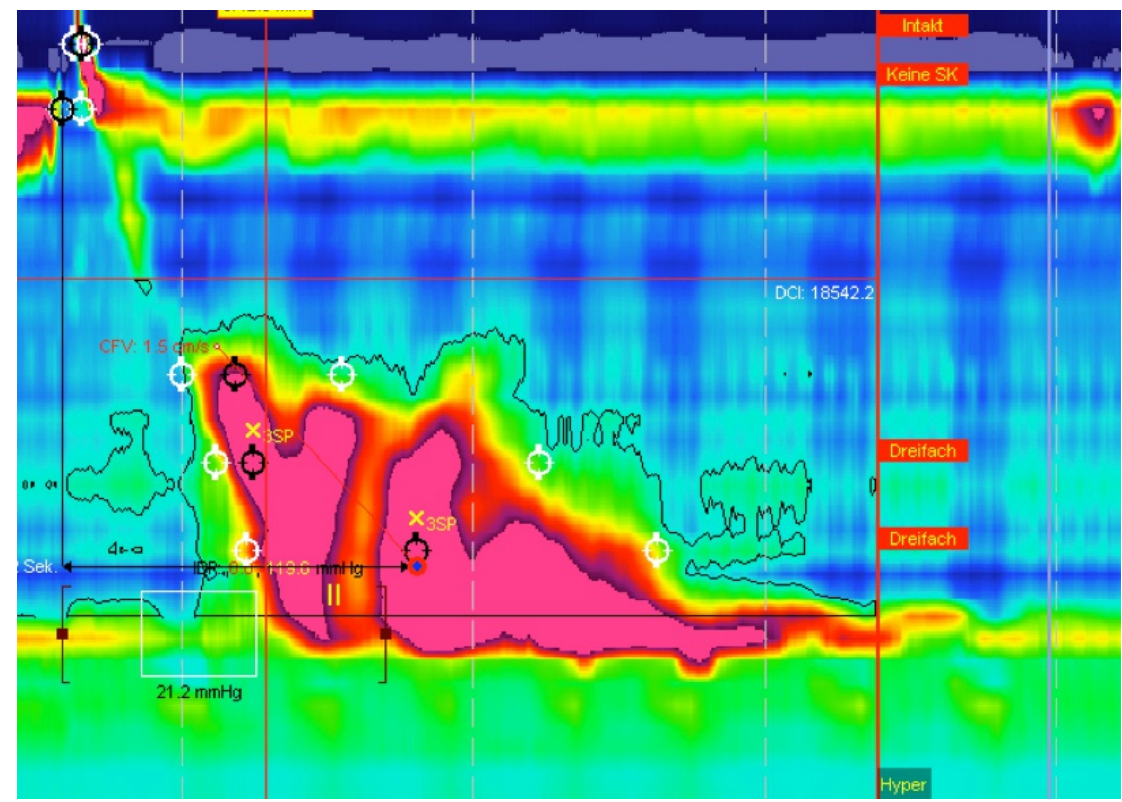

Abb. 3: Achalasie Typ 3 (Quelle: Ordensklinikum Linz) 


\subsection{2. Ösophagogastroduodenoskopie}

Patienten mit Dysphagie Symptomatik bedürfen einer Ösophagogastroduodenoskopie (ÖGD) um zu Beginn eine Obstruktion in Folge einer Tumorerkrankung bzw. eine Pseudoachalasie auszuschließen. Die Befunde einer ÖGD bei Achalasie sind vielfältig und sind abhängig von der individuellen Ausprägung der Erkrankung. Von einer fast unauffälligen Darstellung bis hin zu einem sigmoid-förmigen Megaösophagus ist alles möglich. Typisch sind jedoch Dilatation, Chymus-Retention, Mukosaverdickung und unphysiologische Kontraktionen des Ösophagus, zusammen mit einer funktionalen Stenose des unteren Schließmuskels. Weiße Beläge durch Candidiasis bei immunkompetenten Patienten deuten auf eine ösophageale Stase hin und sind somit ein Hinweis für das Vorliegen einer Motilitätsstörung. Die Passage des Endoskops durch den UÖS erfolgt bei idiopathischer Achalasie typischer Weise gegen einen leichten Widerstand. ${ }^{3,27}$

\subsubsection{Videokinematographie}

Bei dieser Methode wird ein Kontrastmittel-Bolus geschluckt und der Schluckakt mittels Röntgen-Videosequenz festgehalten.

Hinweisend auf eine Achalasie-Erkrankung ist eine signifikante Ösophagusdilatation. Auch Aperistaltik, eine Engstelle im Bereich des ösophagogastralen Übergangs und eine verzögerte Kontrastmittel-Clearance sind typisch.

Die Methode ist u.a. im Rahmen der Therapiekontrolle von Nutzen. Vor allem vor dem Hintergrund, dass das Nachlassen der Symptomatik nicht immer gleichbedeutend mit einer Verbesserung der ösophagealen Clearance ist. ${ }^{3}$

\subsection{Klinik: Eckardt Score}

Um die Symptomatik klinisch zu quantifizieren gibt es den Eckardt Score. Er berücksichtigt die vier Symptome Dysphagie, Regurgitation, Brustschmerz und Gewichtsverlust, die jeweils einzeln nach ihrem Schweregrad beurteilt und mit Punkten versehen werden (siehe Tabelle 1). So ergibt sich ein Score von maximal 12 Punkten. In vorliegender Studie wird der Eckardt Score vor und nach der POEM bestimmt, sodass es bei gutem Therapieansprechen zu einer Senkung des Eckardt Scores kommt, was gleichbedeutend mit einer Besserung der Symptomatik ist. In der Fachliteratur wird ein initialer ES $\leq 3$, der je nach Quelle sechs bis zwölf Wochen postinterventionell erhoben wurde, als Therapieerfolg gewertet. ${ }^{4}$ 


\begin{tabular}{|c|c|c|c|c|}
\hline Symptome & $\begin{array}{c}\text { Bei jeder } \\
\text { Nahrungs- } \\
\text { aufnahme }\end{array}$ & Täglich & Wöchentlich & $\begin{array}{c}\text { Nicht } \\
\text { aufgetreten }\end{array}$ \\
\hline Dysphagie & 3 & 2 & 1 & 0 \\
\hline Regurgitation & 3 & 2 & 1 & 0 \\
\hline $\begin{array}{c}\text { Brustschmerzen } \\
\text { Gewichts- } \\
\text { verlust }\end{array}$ & 3 & 2 & 1 & 0 \\
& 3 & $5-10 k g$ & $<5 k g$ & $\begin{array}{c}\text { Nicht } \\
\text { aufgetreten }\end{array}$ \\
\hline
\end{tabular}

Tab. 1: Eckardt Score (eigene Darstellung)

\subsection{Weitere Therapieformen}

Bis jetzt konnte sich keine Therapie der Achalasie als Standard etablieren, sodass mehrere Optionen existieren, die individuell und situativ eingesetzt werden.

Alle Methoden sollen die Relaxation des unteren Ösophagus verbessern, um eine weitgehend normale Nahrungsaufnahme zu ermöglichen.

\subsubsection{Pharmakotherapie}

Die Pharmakotherapie ist die am wenigsten effektive Behandlungsmöglichkeit, findet jedoch Anwendung bei Patienten, die aus gesundheitlichen Gründen keine Operation tolerieren, sich gegen einen Eingriff entschieden haben oder ganz milde Symptome aufweisen.

Die häufigsten verwendeten Substanzen sind Nitrate und Calcium-Kanal-Blocker. Ihre Wirkung besteht in der Relaxation der glatten Muskulatur des unteren Sphinkters. Als Wirkstoffe werden meist Isosorbiddinitrat (Nitrat) und Nifedipin (Ca-Kanal-Blocker) eingesetzt. Die gängige Applikationsart ist sublingual. Beispielsweise werden $5 \mathrm{mg}$ Isosorbiddinitrat jeweils 10-15 Minuten jeweils vor den Mahlzeiten eingenommen. ${ }^{28}$ Die Arzneimittel senken den Druck im UÖS ungefähr auf die Hälfte ab. In Studien zeigte das Nitrat eine Besserung der Symptomatik in 53-87\% der Fälle, während Nifedipin eine Symptomverbesserung in $0-75 \%$ der Fälle verzeichnete. ${ }^{29}$

Die Wirkung der Arzneimittel hält nicht lange an, sodass Symptome nur für eine relativ kurze Zeit nach der Applikation verbessert werden können. Die Verwendung wird zusätzlich von der auftretenden Tachyphylaxie und den bei $30 \%$ der Patienten auftretenden Nebenwirkungen limitiert. ${ }^{29}$ 


\subsubsection{Botulinumtoxin}

Die Therapie mit Botulinumtoxin (BT) ist eine sichere Methode mit sehr wenigen Komplikationen. Deshalb eignet sie sich v.a. für ältere oder multimorbide Patienten. Die Injektion erfolgt im Rahmen einer Routine-Endoskopie. Dabei wird der distale Sphinkter in vier Quadranten eingeteilt, in welche jeweils eine BT-Injektion ca. einen Zentimeter über der Z-Linie verabreicht wird. ${ }^{30}$

Botulinumtoxin A reduziert den Tonus des UÖS, indem es die Acetylcholin-Sekretion von cholinergen Neuronen im Plexus Myentericus blockiert. ${ }^{30}$

Das Ansprechen auf eine Therapie mit BT-Injektionen liegt bei Achalasie Patienten über $80 \%$ nach einem Monat. Meist tritt die Wirkung sogar bereits nach $24 \mathrm{~h}$ ein. Der Nachteil dieser Therapie liegt in ihrer rasch absinkenden Wirkung. Weniger als $60 \%$ der Behandelten befinden sich nach einem Jahr noch in Remission. Daher ist es oft nötig, die Therapie bei Verschlechterung der Beschwerden zu wiederholen. ${ }^{31}$

\subsubsection{Pneumatische Ballondilatation}

Bei der Therapie mittels Pneumatischer Ballondilatation (PD) wird ein Ballon, der anfangs noch keine Luft enthält, entlang eines Führungsdrahts eingeführt und auf Höhe des kontrahierten unteren Sphinkters aufgeblasen. Für den therapeutischen Erfolg ist hauptsächlich die Dehnung der glatten Muskulatur, weniger -wie häufig angenommen- die Ruptur von Muskelfasern, verantwortlich. ${ }^{32}$

Der Eingriff wird meist in gewissen Abständen wiederholt. Dabei wird jedes Mal ein größerer Ballon verwendet und somit eine größere Dehnung erzielt. Initial nach dem Eingriff liegt die Erfolgsrate im Hinblick auf die Symptomremission bei über 85\%, welche auch mittelfristig (12-24 Monate) aufrecht erhalten bleibt. ${ }^{33}$ Nach fünf Jahren kommt es bei etwas mehr als der Hälfte der Patienten (56\%) zu erneutem Auftreten der Symptome und sie bedürfen eines erneuten Eingriffs. ${ }^{34}$ Deshalb ist es empfehlenswert, behandelte Patienten regelmäßig mittels Videokinematographie zu kontrollieren, um solche Rückfälle frühzeitig zu erkennen und möglicherweise eine erneute PD zu veranlassen. ${ }^{35}$

Ein Nachteil der pneumatischen Ballondilatation ist das Risiko einer Ösophagusperforation. In einer multizentrischen randomisierten Studie von Boeckxstaens et. al. wurden 95 Patienten mittels PD behandelt, bei vier Patienten $(4 \%)$ ist eine Perforation aufgetreten. ${ }^{33}$

\subsubsection{Laparoskopische Heller Myotomie}

Die Laparoskopische Myotomie nach Heller (LHM) ist zusammen mit der PD die derzeit gebräuchlichste Methode zur Behandlung der Achalasie.

Diese Therapie der Myotomie wurde erstmals vom Namensgeber Ernst Heller 1913 durchgeführt und beschrieben. Sie entwickelte sich in den letzten Jahren zu einem laparoskopischen Eingriff. Der therapeutische Effekt beruht auf dem Durchtrennen von Muskelfasern des UÖS, dessen pathologisch erhöhter Tonus dadurch gesenkt werden kann.

Der Invasivität der Methode geschuldet, gibt es einige Kriterien, die Patienten erfüllen sollten, bevor sie mit der LHM behandelt werden können. So spielen beim Auswahlprozess Faktoren wie Alter, Bildgebung, frühere Operationen, Schwere und Dauer der Symptomatik, sowie Komorbiditäten eine Rolle. ${ }^{36}$ 
Eine Besserung der Symptome ist initial bei ca. 90\% der operierten Patienten vorhanden. Mittelfristig ergeben sich ähnliche Tendenzen wie bei anderen Therapiemethoden. ${ }^{37}$ Das Risiko fünf Jahre nach einer Therapie ein Rezidiv zu entwickeln liegt bei $30 \%$ und steigt nach zehn Jahren auf $37 \%$ an. ${ }^{34}$

Aufgrund der häufig auftretenden post-operativen Reflux-Symptomatik wird bei den meisten LHM zusätzlich eine Fundoplicatio durchgeführt. Bei dieser Operation wird der Magenfundus wie eine Manschette um den Ösophagus gelegt. Die Fundoplicatio ist ein Routineeingriff, durch den das Refluxrisiko signifikant verringert werden kann. 


\section{Abstrakt}

\section{Englisch}

Introduction Peroral endoscopic myotomy is a minimal invasive technique for the treatment of achalasia. We analyzed the clinical outcome of POEM at Ordensklinikum Linz to evaluate its safety and efficacy.

Methods We retrospectively collected data from patients with achalasia who have been treated with POEM at the Ordensklinikum Linz between January 2015 and August 2018. Preoperative assessment included HR-manometry, gastroscopy, videocinematography and clinical evaluation. Postoperative assessment was performed 6 weeks after treatment including gastroscopy and clinical reevaluation.

Results 43 patients underwent POEM. 22 (51.2\%) were women and 21 (48.8\%) were men. The mean age was 55.1 years, ranging from 17 to 84 years. Previous interventions included Botox injection in 6 patients (13.9\%) and pneumatic balloon dilatation in 12 patients $(27.9 \%)$. According to Chicago classification 30 patients (85.7\%) were diagnosed with type II achalasia, 4 patients $(11.4 \%)$ with type III achalasia and 2 patients $(5.7 \%)$ with type I achalasia. The mean length of myotomy was $8.5 \mathrm{~cm} \pm 0.5$. Myotomy was extended for $2 \mathrm{~cm}$ beyond the gastroesophageal junction. From 36 evaluable interventions, POEM was successfully performed (defined by Eckardt score $\leq 3)$ in 33 cases $(91,7 \%)$. 3 patients showed relapses $(8,3 \%)$. Overall Eckardt score could be reduced significantly from $7.0 \pm 0.3$ to $1.0 \pm 0.2$ after POEM $(p<0,0001)$. We observed 5 adverse events (11.6\%), all of them could be managed conservatively. Regarding postoperative reflux disease, 24 patients could be evaluated. 9 patients $(37,5 \%)$ were diagnosed with erosive esophagitis or symptomatic reflux disease.

7 patients were endoscopically diagnosed with erosive esophagitis grade A $(20,8 \%)$ and $B$ $(8,3 \%)$ according to Los Angeles classification. Another 2 patients showed signs of symptomatic reflux disease.

Conclusion POEM is a safe and effective procedure to achieve symptom relief in achalasia patients. More studies and data are needed in terms of long-term outcome of effectiveness and development of postinterventional reflux disease. 


\section{Deutsch}

Einleitung Die perorale endoskopische Myotomie ist ein minimalinvasives Verfahren zur Therapie der Achalasie. In dieser Studie analysieren wir das Outcome der POEM am Ordensklinikum Linz, um die Sicherheit und Effektivität der Methode zu evaluieren.

Methodik Unsere retrospektive Datenanalyse umfasst alle Achalasie Patienten, die von Januar 2015 bis August 2018 am Ordensklinikum Linz mittels POEM behandelt wurden. Die präoperative Evaluierung beinhaltet die Durchführung einer HR-Manometrie, ÖGD, Videokinematographie und eine Evaluierung der klinischen Beschwerden der Patienten. Nachkontrollen wurden 6 Wochen nach POEM in Form einer Kontrollgastroskopie und ReEvaluierung der klinischen Beschwerden durchgeführt.

Ergebnisse Im Rahmen der Studie wurden 43 Patienten mit POEM behandelt. $22(51,8 \%)$ waren Frauen, 21 (48,8\%) waren Männer. Das durchschnittliche Alter betrug 55,1 Jahre und reichte von 17 bis 84 Jahren. 6 Patienten (13,9\%) waren mit Botoxinjektion, 12 Patienten $(27,9 \%)$ mit pneumatischer Ballondilatation vorbehandelt worden. Entsprechend der Chicago Klassifikation konnten 30 Patienten (85.7\%) mit einer Achalasie Typ II, 4 Patienten (11.4\%) mit einer Achalasie Typ III und 2 Patienten (5.7\%) mit einer Achalasie Typ I diagnostiziert werden. Die mittlere Länge der Myotomie betrug $8.5 \mathrm{~cm} \pm 0.5$. Die Myotomie erstreckte sich bis $2 \mathrm{~cm}$ über den gastroösophagealen Übergang hinaus. Von 36 auswertbaren Eingriffen wurde in 33 Fällen (91,7\%) ein initialer Therapieerfolg (definiert als Eckardt Score $\leq 3$ ) erzielt. 3 Patienten $(8,3 \%)$ entwickelten ein Rezidiv. Insgesamt konnte eine signifikante Reduktion des Eckardt Scores von $7.0 \pm 0.3$ auf $1.0 \pm 0.2$ nach POEM erreicht werden $(p<0,0001)$. Wir beobachteten 5 Komplikationen (11.4\%), welche konservativ behandelt werden konnten. Bezüglich postoperativ neu aufgetretener Refluxerkrankungen konnten 24 Datensätze ausgewertet werden. 9 Patienten (37,5\%) zeigten eine erosive Ösophagitis oder eine symptomatische Refluxerkrankung. Bei 7 Patienten wurde endoskopisch nach der Los Angeles Klassifikation eine erosive Ösophagitis Grad A $(20,8 \%)$ und Grad B $(8,3 \%)$ diagnostiziert. Weitere 2 Patienten zeigten typische Symptome einer symptomatischen Refluxerkrankung.

Fazit Die perorale endoskopische Myotomie ist eine effektive und sichere Methode zur Therapie der Achalasie. Um die langfristige Effektivität der POEM und das Auftreten postinterventioneller Refluxerkrankungen zu evaluieren, bedarf es weiterer Studien. 


\section{Zielsetzung}

In vorliegender retrospektiver Studie soll der Stellenwert der POEM zur Behandlung der Achalasie am Ordensklinikum Linz ermittelt werden. Es werden Therapieerfolg und Komplikationsraten untersucht. Ein weiteres Augenmerk gilt der Frage, ob bei Therapie mittels POEM postinterventionell ein erhöhtes Risiko für eine gastroösophageale Refluxkrankheit besteht. Die Studie leistet auf diese Weise einen wichtigen Beitrag zur Qualitätssicherung im Bereich der Achalasie Therapie.

Die erhobenen Studiendaten sollen mit der aktuellen Studienlage verglichen werden, um Aussagen speziell für das Ordensklinikum Linz treffen zu können.

Zudem soll die Arbeit als Grundlage für künftige Studien dienen. 


\section{Material und Methodik}

\subsection{Statistische Methoden}

Es handelt sich um eine rein deskriptive Studie. Zur statistischen Auswertung werden Mittelwerte (mean) und Standardfehler (SEM - standard error oft the mean) angegeben. Die Datenanalyse erfolgt mittels Microsoft Excel.

\subsection{Patientenkollektiv}

Es wurden insgesamt 46 Patienten in einem Zeitraum von 01.01.2015 bis 01.08.2018 einer Therapie mittels POEM zugeführt und somit erfasst. Zwei Patientinnen erhielten eine POEM bei Jackhammer-Ösophagus. Da unsere Studie sich auf die POEM bei Achalasie Patienten beschränkt, werden sie aus der Studie ausgeschlossen. Eine Achalasie Patientin zeigte endoskopisch zu Beginn der POEM eine stark vulnerable Ösophagusmukosa, sodass eine POEM aufgrund des zu hohen Blutungsrisikos nicht sinnvoll erschien. Die POEM wurde abgebrochen, daher wird die Patientin aus der Studie ausgeschlossen. Es konnten daher 43 Patienten in die Studie eingeschlossen werden.

\subsection{Perorale endoskopischer Myotomie}

\subsubsection{Präinterventionelle Diagnostik}

Die Achalasie Patienten der Studie wurden mittels HR-Manometrie, Ösophagogastroduodenoskopie, Videokinematographie und bildgebenden Verfahren diagnostiziert.

Für eine POEM ausgewählte Patienten nehmen 24-48h vor dem Eingriff lediglich klare Flüssigkeiten zu sich. In diesem Zeitraum erfolgt eine Gastroskopie, um eine Stase von Nahrungsresten bzw. eine Soor-Infektion auszuschließen. Am Tag des Eingriffs wird eine Antibiose-Prophylaxe verabreicht, die für die Dauer des Aufenthalt weitergeführt wird und in Einzelfällen bis auf sieben Tage verlängert werden kann. ${ }^{38}$

\subsubsection{Operative Vorgehensweise}

\subsubsection{Submukosadissektion}

Die POEM wird in Intubationsnarkose durchgeführt. Die Endoskopie erfolgt mit aufgesetzter Distanzkappe und unter CO2-Insufflation.

Zu Beginn werden anatomische Landmarken ausgemessen (oberer und unterer Ösophagussphinkter). Nun wird die Inzisionsstelle auf Höhe des Aortenbogens und des rechten Hauptbronchus an der anterioren Wand des Ösophagus gewählt. Zunächst wird die Mukosa mit $10 \mathrm{ml}$ mit $\mathrm{NaCl} 0,9 \%$ verdünntem Indigokarmin unterspritzt und ein submukosales Depot geschaffen. Die zwei Zentimeter lange Mukosainzision erfolgt mittels Triangle Tip Knife ${ }^{\circledR}$ (Olympus). Anschließend wird die Submukosa unter wiederholter Injektion von Indigokarminverdünntem $\mathrm{NaCl}$ kaudalwärts disseziert. Am 
gastroösophagealen Übergang ändert sich die Faserstruktur. Die zirkulären Muskelfasern des Ösophagus treffen auf die irregulär angeordneten Muskelfasern der Kardia des Magens. Zur Lagekontrolle wird im Magen in Inversion sichergestellt, dass der Tunnel etwa zwei Zentimeter distal des gastroösophagealen Übergangs zu Liegen kommt. ${ }^{1,38}$

\subsubsection{Myotomie}

Die Myotomie der zirkulären Fasern wird ca. drei Zentimeter distal der Inzisionsstelle begonnen. Die longitudinalen Muskelfasern kommen zum Vorschein und werden verschont. Sie dienen als Sicherheitsschicht um eine Perforation in das Mediastinum bzw. Peritoneum zu vermeiden. Die Myotomie wird nach distal zwei Zentimeter über den gastroösophagealen Übergang hinaus fortgeführt. Zur Blutstillung wird eine Koagulationszange (Coagrasper ${ }^{\circledR}$, Olympus) verwendet.

Nach vollendeter Myotomie wird die nun verbesserte Passage durch den UÖS überprüft. Es erfolgt eine abschließende Kontrolle auf Bluttrockenheit im submukosalen Tunnel.

Die Mukosainzision wird mit Endoclips verschlossen. ${ }^{1,38}$

\subsubsection{Postoperative Betreuung}

Postoperativ wird eine insgesamt sechs wöchige PPI-Therapie begonnen. Diese wird im Rahmen des stationären Aufenthalts intravenös appliziert, mit Oralisierung bei Entlassung. In den ersten beiden postoperativen Wochen wird Breikost angeordnet. Am ersten postinterventionellen Tag wird eine Videokinematographie erstellt, um eventuelle Leaks frühzeitig zu erkennen. Treten keine Komplikationen auf, werden die Patienten innerhalb von drei Tagen nach POEM entlassen. ${ }^{38}$

Die Nachkontrollen wurden ca. sechs Wochen post POEM in Form einer ÖGD durchgeführt. Im selben Rahmen wurde der postinterventionelle Eckardt Score erhoben. Die erste Nachkontrolle wurde Ende Februar 2015 durchgeführt. 


\section{Ergebnisse}

\subsection{Patientencharakteristika}

Im Zeitraum von 01.01.2015 bis 01.08.2018 erfolgte bei 43 Patienten eine POEM zur Therapie der Achalasie. Davon waren 22 (51\%) weiblichen und 21 (49\%) männlichen Geschlechts.

Das Patientenalter zum Zeitpunkt des Eingriffs lag bei den Frauen zwischen 18 und 84 Jahren (Mittelwert $58 \pm 4,1$ Jahre) und bei den Männern zwischen 17 und 82 Jahren (Mittelwert $52 \pm 4,1$ Jahre).

Die Achalasie Typ II war mit 85,7\% die häufigste Form der Achalasie. Typ I (5,7\%) und Typ III $(11,4 \%)$ waren seltener vertreten. Aufgrund der geringen Anzahl an Patienten mit Achalasie Typ I und III wird auf einen Vergleich der einzelnen Achalasie Typen verzichtet. Bei 7 Patienten lag kein manometrischer Befund in unserer Datenbank vor (s. Tabelle 2). 
- Geschlecht

$$
\begin{array}{cl}
\text { Frauen, } n(\%) & 22(51,2) \\
\text { Männer, } n(\%) & 21(48,8) \\
\text { - } \quad \text { Alter, Jahr (mean } \pm \text { SEM) } & 55,1 \pm 3,0 \\
\text { Frauen (mean } \pm \text { SEM) } & 58 \pm 4,2 \\
\text { Männer (mean } \pm \text { SEM) } & 52,1 \pm 4,1
\end{array}
$$

- Präinterventioneller Eckardt Score (mean $\pm \quad 7,0 \pm 0,3$
SEM)

Vorhanden, $n(\%) \quad 33(76,7)$

Nicht vorhanden, $n(\%) \quad 10(23,3)$

Vorangegangene Interventionen

Botoxinjektion, $n(\%) \quad 6(13,9)$

Ballondilatation, $n(\%) \quad 12(27,9)$

Keine, $n(\%) \quad 25(58,1)$

Präinterventionelle Evaluierung

- Manometrie

Chicago Klassifikation

$\begin{array}{cl}\text { Achalasie Typ I, } n \text { (\%) } & 2(5,7) \\ \text { Achalasie Typ II, } n(\%) & 30(85,7) \\ \text { Achalasie Typ III, } n(\%) & 4(11,4) \\ \text { Nicht vorhanden, } n(\%) & 7(20) \\ \text { IRP, mmHg (mean } \pm \text { SEM) } & 25,7 \pm 1,8 \\ \text { Vorhanden, } n(\%) & 31(72,1) \\ \text { Nicht vorhanden, } n(\%) & 12(27,9)\end{array}$

Interventionelle Parameter

- Myotomielänge, cm (mean \pm SEM) $\quad 8,5 \pm 0,5$ 
In den folgenden Abschnitten werden die Ergebnisse in Zusammenhang mit den Altersgruppen, den klinischen Beschwerden, d.h. dem präinterventionellen Eckardt Score und den IRP Werten analysiert.

Die Altersgruppen (AG) werden wie folgt eingeteilt.

Das Alter bezieht sich dabei auf den Zeitpunkt des Eingriffs.

Anzahl $n$ (mittleres Alter \pm SEM)

\begin{tabular}{ll}
\hline Altersgruppe 1: 15-30 Jahre & $6(19,8 \pm 0,9)$ \\
\hline Altersgruppe 2: 31-45 Jahre & $7(39,0 \pm 0,9)$ \\
\hline Altersgruppe 3: 46-60 Jahre & $10(53,9 \pm 1,0)$ \\
\hline Altersgruppe 4: 61-75 Jahre & $13(67,5 \pm 1,2)$ \\
\hline Altersgruppe 5: 76-90 Jahre & $7(80,4 \pm 0,8)$ \\
\hline
\end{tabular}

Tab. 3: Altersgruppen

\subsection{Outcome}

In unserer Studie erfolgte ca. sechs Wochen nach POEM ein Follow-Up mittels ÖGD. Im Rahmen dieser Kontrolle wurde der postinterventionelle Eckardt Score erhoben.

Von 36 auswertbaren Datensätzen konnten 33 Patienten $(91,7 \%)$ erfolgreich mit POEM behandelt werden, definiert als Eckardt Score $\leq 3$. Sieben Datensätze zum postinterventionellen Follow-Up fehlen.

Der Eckardt Score unseres Patientenkollektivs konnte 6 Wochen nach der POEM von durchschnittlich 7,0 \pm 0,3 Punkten auf 1,0 \pm 0,2. Punkte gesenkt werden. Das Ergebnis ist statistisch höchst signifikant $(p<0,0001)$.

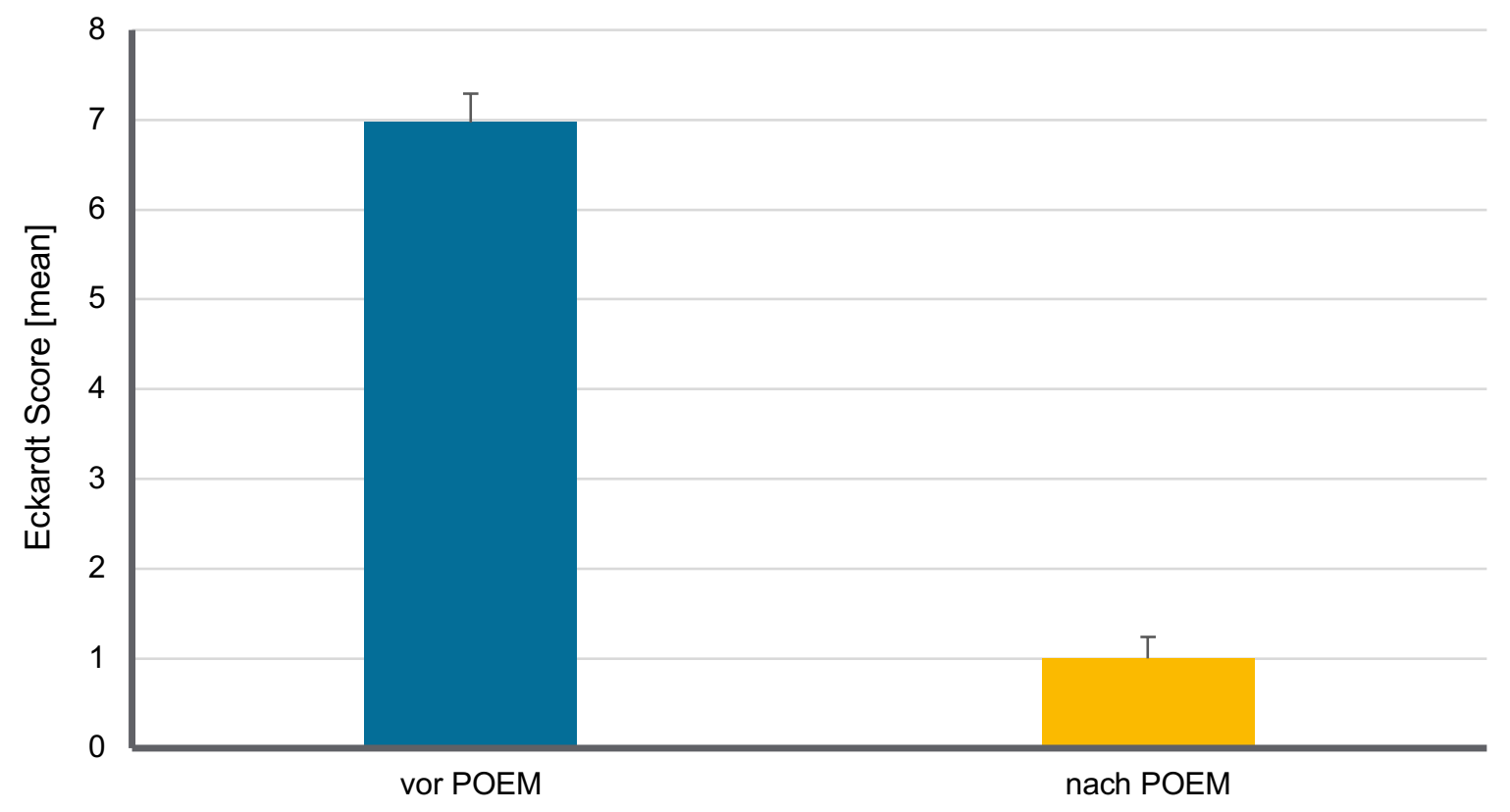

Abb. 4: Durchschnittlicher Eckardt Score vor und nach POEM 
In nachfolgendem Diagramm ist die Verteilung des prä- und postinterventionellen ES aufgeführt.

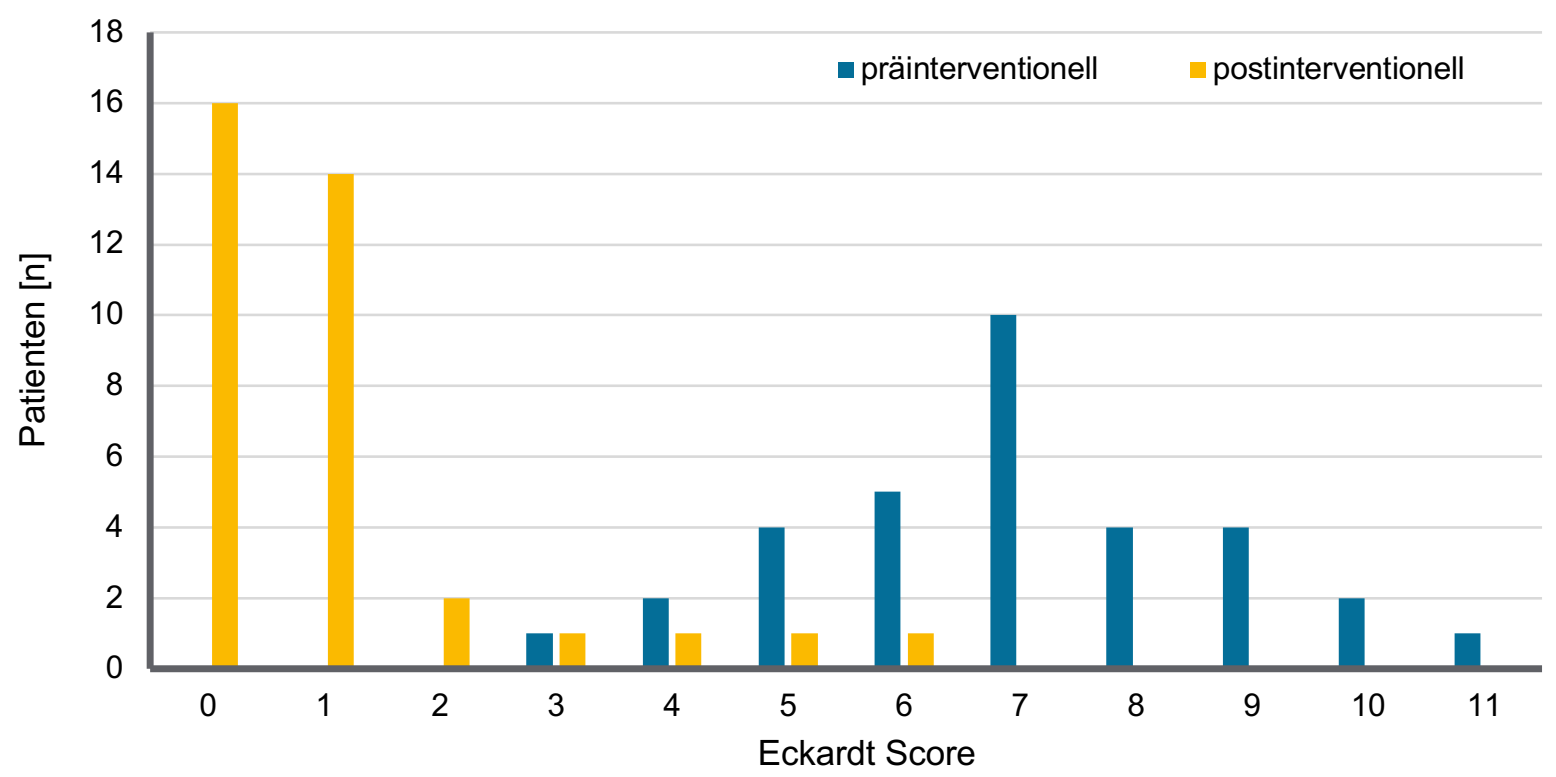

Abb. 5: Verteilung der prä- und postinterventionellen ES

Zum Zeitpunkt der Reevaluation nach 6 Wochen wurden drei Patienten mit einem Rezidiv $(E S>3)$ beobachtet.

\subsection{Postinterventioneller Reflux}

Die Evaluierung der potentiell POEM-induzierten postinterventionellen Refluxerkrankungen erfolgte durch Kontroll-Gastroskopien und/oder entsprechende typische Klinik der Patienten (Sodbrennen) im Verlauf.

Nach 43 durchgeführten Interventionen konnten zur Auswertung der postinterventionell entwickelten Refluxbeschwerden die Daten von 24 Patienten $(55,8 \%)$ herangezogen werden.

Von insgesamt 24 auswertbaren Datensätzen wurden 9 Fälle (37,5\%) von neu aufgetretener erosiver Refluxkrankheit oder symptomatischer Refluxösophagitis beobachtet. Der Diagnosezeitpunkt bezogen auf die POEM reicht bei diesen Patienten von sechs Wochen (im Rahmen der standardmäßigen Kontroll-ÖGD) bis hin zu acht Monaten postinterventionell. 


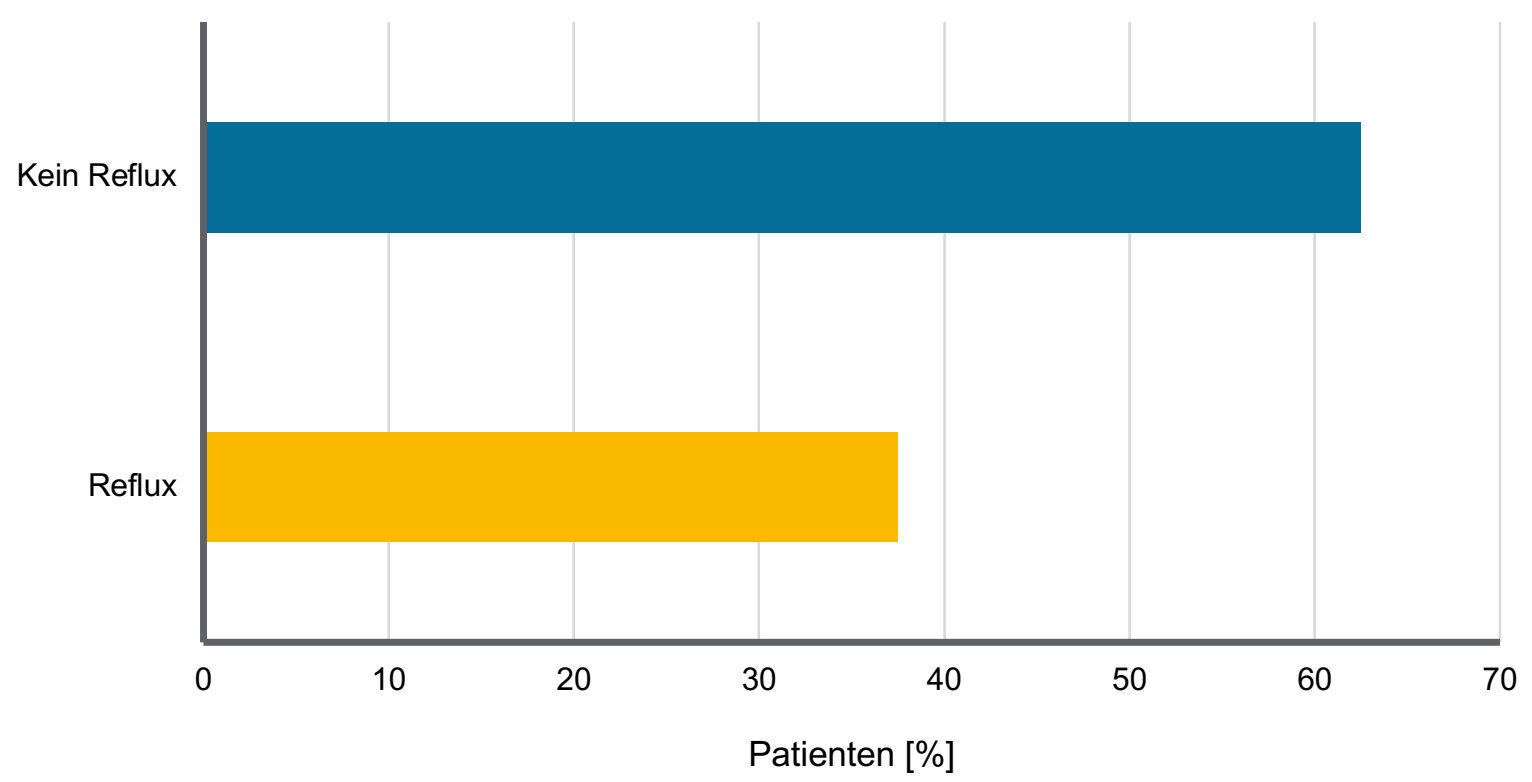

Abb. 6: Refluxentwicklung nach POEM

Zwei Patienten (8,3\%) gaben im Verlauf eine typische Klinik bzw. neu aufgetretenes Sodbrennen an. Ein endoskopischer Befund dieser Patienten zum Zeitpunkt der Beschwerden liegt nicht vor. Bei 7 Studienteilnehmern erfolgt eine endoskopische Diagnose einer erosiven Refluxkrankheit (ERD) im Sinne der Los Angeles Klassifikation. Bei fünf Patienten (20,8\%) handelt es um eine ERD Los Angeles Grad A, bei zwei Patienten $(8,3 \%)$ um eine ERD Los Angeles Grad B.

In unten angeführter Tabelle ist die Los Angeles Klassifikation der Refluxösophagitis aufgeschlüsselt. ${ }^{39}$

\begin{tabular}{ll}
\hline Grad & Befundmerkmale \\
\hline A & Eine oder mehrere Erosionen von maximal fünf Millimetern Länge \\
\hline B & Eine oder mehrere Erosionen auf den Faltenkuppen, davon eine $>$ fünf \\
& Millimeter \\
C & Erosionen, die sich über mehrere Faltenkuppen erstrecken, aber maximal \\
& $75 \%$ der Zirkumferenz betreffen \\
\hline & $>75 \%$ der Zirkumferenz reichende Erosionen \\
\hline
\end{tabular}

Tab. 4: Los Angeles Klassifikation der Refluxösophagitis (Quelle: Rath et al., Gastrointestinal Endoscopy, 2004) 


\subsubsection{Postinterventionelle Refluxkrankheit bezogen auf das Patientenalter}

Untenstehendes Diagramm stellt die postinterventionelle Refluxkrankheit nach Altersgruppe geordnet dar.

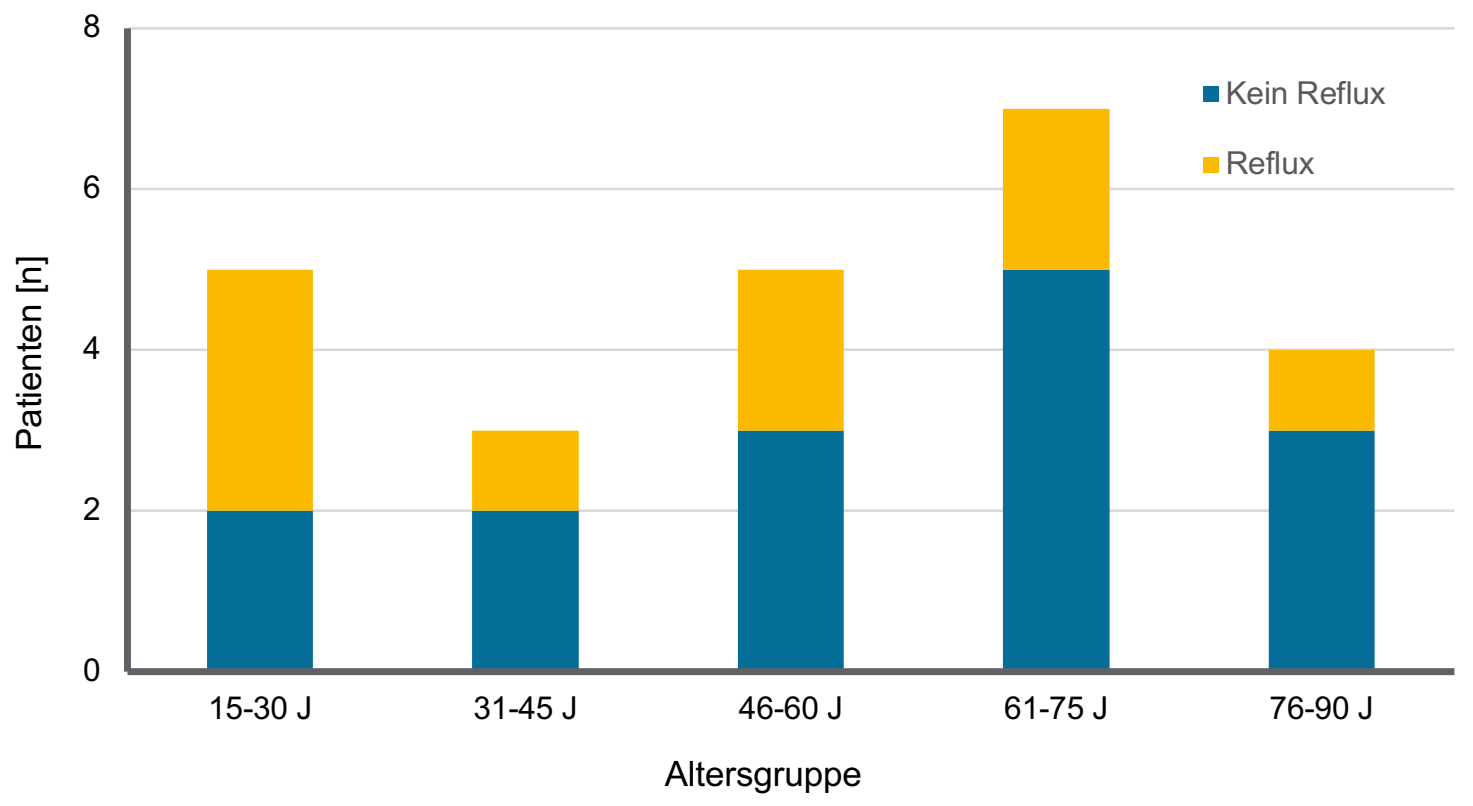

Abb. 7: Refluxentwicklung nach Altersgruppe

Das Entstehen von postinterventionellen Refluxbeschwerden zieht sich durch alle Altersgruppen, in unserer Studie lassen sich jedoch aufgrund der niedrigen Fallzahlen bzw. fehlenden Datensätze keine weiteren Tendenzen erkennen.

\subsubsection{Postinterventionelle Refluxkrankheit in Zusammenhang mit dem präinterventionellen IRP-Wert}

Folgende Graphik zeigt die Verteilung der präinterventionellen IRP Werte in aufsteigender Reihenfolge. Gelb hervorgehobene Säulen sprechen für eine postinterventionell neu aufgetretene Refluxkrankheit.

Es konnten 17 von 43 Datensätzen ausgewertet werden. 


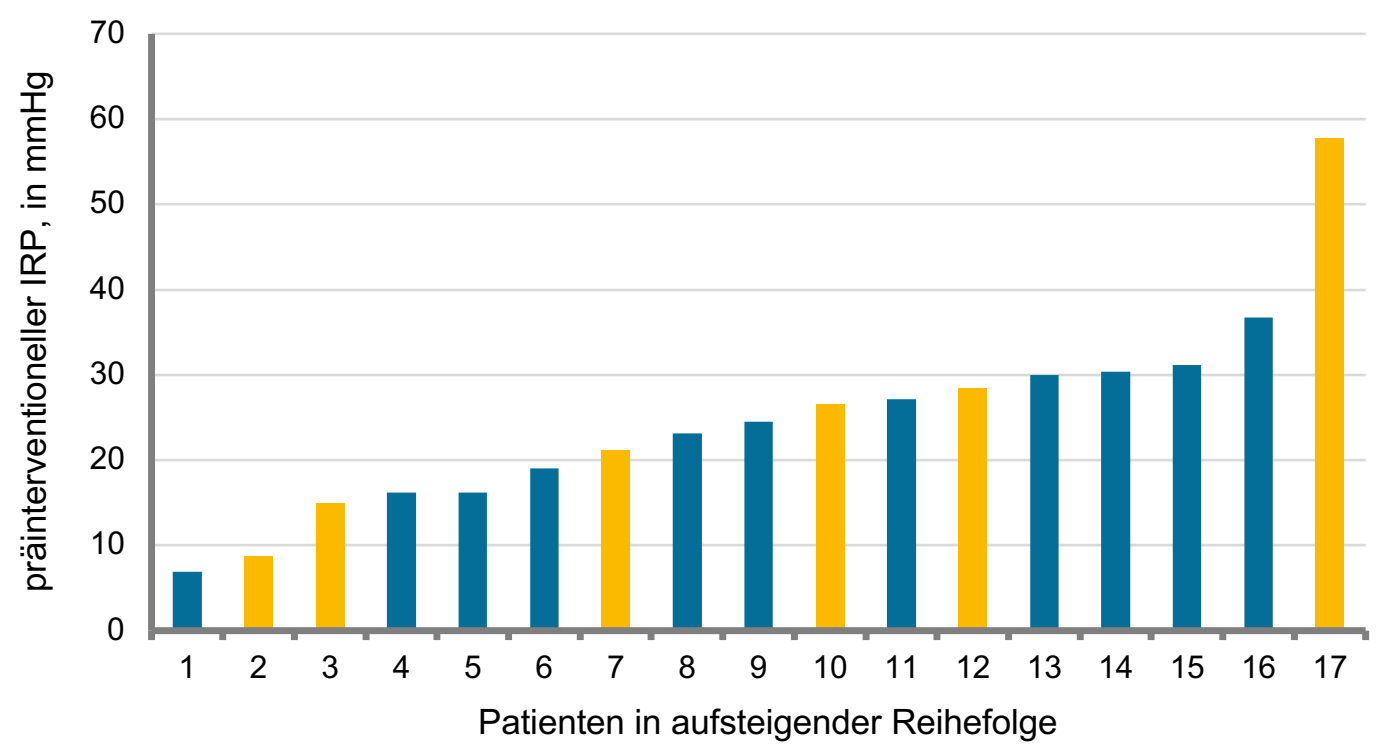

Abb. 8: Verteilung des IRP im Zusammenhang mit Refluxkrankheit nach POEM

Patienten mit Refluxbeschwerden sind nahezu gleichmäßig auf alle IRP-Werte verteilt. Aus unseren Datensätzen lässt sich keine Korrelation zwischen präinterventionellem IRP und dem Auftreten einer Refluxkrankheit nach POEM herstellen.

\subsection{Komplikationen}

Von 43 durchgeführten POEMs traten bei fünf Patienten Komplikationen auf (11,6\%).

Dabei handelt es sich um zwei arterielle Blutungen, davon eine transfusionspflichtig.

Eine Studienteilnehmerin mit Pneumonie wurde antibiotisch therapiert.

Eine Patientin mit Pneumothorax wurde vier Tage auf der Intensivstation überwacht. Die Anlage einer Thoraxdrainage war nicht erforderlich. Dies resultierte in einem verlängerten Gesamtklinikaufenthalt von 7 Tagen.

Bei einer Patientin mit Thorax- und Halsemphysem sowie Unterlappenatelektasen beidseits erfolgte eine fünftägige Observanz auf Intensivstation. Die Patientin konnte am ersten postoperativen Tag extubiert werden. Der Gesamtklinikaufenthalt der Patientin betrug hier vier Wochen, die letzten beiden Wochen zur Remobilisation.

Anzahl der Komplikationen, $n(\%)$

\begin{tabular}{ll}
\hline Arterielle Blutung (nicht transfusionspflichtig) & $1(2,3 \%)$ \\
\hline Arterielle Blutung (transfusionspflichtig) & $1(2,3 \%)$ \\
\hline Pneumonie & $1(2,3 \%)$ \\
\hline Pneumothorax & $1(2,3 \%)$ \\
\hline Intraoperatives Thorax- und Halsemphysem, & $1(2,3 \%)$ \\
Unterlappenatelektasen bds. & \\
\hline
\end{tabular}

Tab. 5: Komplikationen 


\subsubsection{Komplikationen nach Altersgruppe}

In untenstehender Tabelle werden die Komplikationsraten der einzelnen Altersgruppen angegeben.

\begin{tabular}{|c|c|}
\hline & Anzahl der Komplikationen, $n(\%)$ \\
\hline AG 1 (15-30 Jahre) & $0(0)$ \\
\hline AG 2 (31-45 Jahre) & $0(0)$ \\
\hline AG 3 (46-60 Jahre) & $1(10)$ \\
\hline $\begin{array}{ll}\text { - } & \text { Arterielle Blutung (nicht } \\
& \text { transfusionspflichtig) }\end{array}$ & $1(10)$ \\
\hline AG 4 (61-75 Jahre) & $2(15,4)$ \\
\hline - Pneumonie & $1(7,7)$ \\
\hline - Pneumothorax & $1(7,7)$ \\
\hline AG 5 (76-90 Jahre) & $2(28,6)$ \\
\hline $\begin{array}{l}\text { - Intraoperatives Thorax- und } \\
\text { Halsemphysem, } \\
\text { Unterlappenatelektasen bds. } \\
\text { - } \quad \text { Arterielle Blutung } \\
\text { (transfusionspflichtig) }\end{array}$ & $1(14,3)$ \\
\hline
\end{tabular}

Tab. 6: Komplikationen nach Altersgruppe

In Altersgruppe 1 und 2 (15-30 und 31-45 Jahre) wurden keine Komplikationen verzeichnet. In Altersgruppe 3 (46-60 Jahre) kam es zu einer nicht transfusionspflichtigen Blutung. Bei den Patienten zwischen 61 und 75 Jahren (AG 4) traten eine Pneumonie und ein Pneumothorax auf.

In Altersgruppe 5 (76-90 Jahre) trat eine transfusionspflichtige Blutung auf. Zudem wurde eine Patientin intensivpflichtig bei Thorax- und Halsemphysem sowie Unterlappenatelektasen beidseits.

Die erhobenen Daten zeigen eine deutliche Tendenz. Es zeigt sich erwartungsgemäß ein proportionaler Zusammenhang zwischen Alter und Komplikationsrate. Der Korrelationskoeffizient beträgt 0,96. Der längste Gesamtkrankenhausaufenthalt ergab sich ebenfalls für die älteste Patientengruppe. 


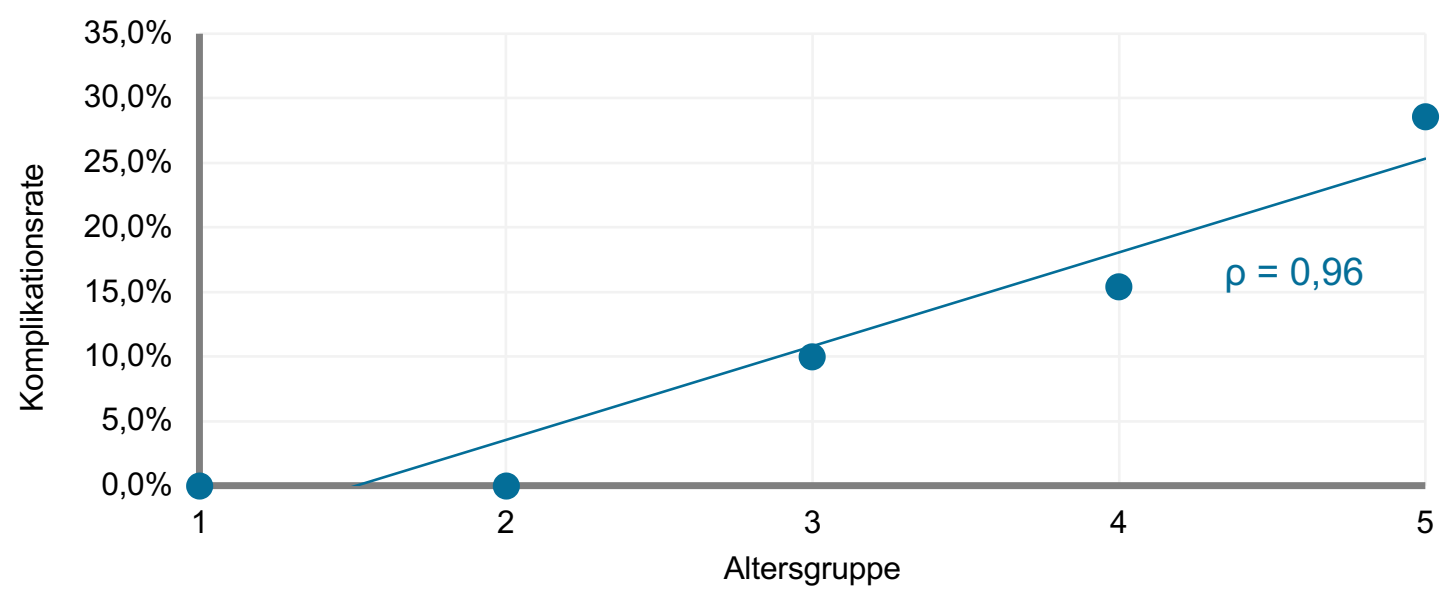

Abb. 9: Komplikationsraten nach Altersgruppe

Darüber hinaus wurde insgesamt zwölfmal ein intraoperativ zu entlastendes Pneumoperitoneum $(27,9 \%)$ beobachtet, davon elf in den höheren Altersgruppen 4 und 5 (61-75 Jahre und 76-90 Jahre). Bei den 61-75 Jahre alten Patienten entwickelten sieben $(53,8 \%)$ ein Pneumoperitoneum, das intraoperativ entlastet werden musste. Bei den Patienten im Alter von $76-90$ Jahren waren es vier $(57,1 \%)$.

\subsection{Rezidiventwicklung}

Insgesamt wurde bei 36 Patienten der postinterventionelle Eckardt Score im Rahmen der Reevaluation nach sechs Wochen erhoben.

Drei Patienten zeigten zum Zeitpunkt der Kontrolluntersuchung ein Rezidiv, definiert als Eckardt Score $>3$. Dadurch ergibt sich eine Rezidivrate von 8,3\%.

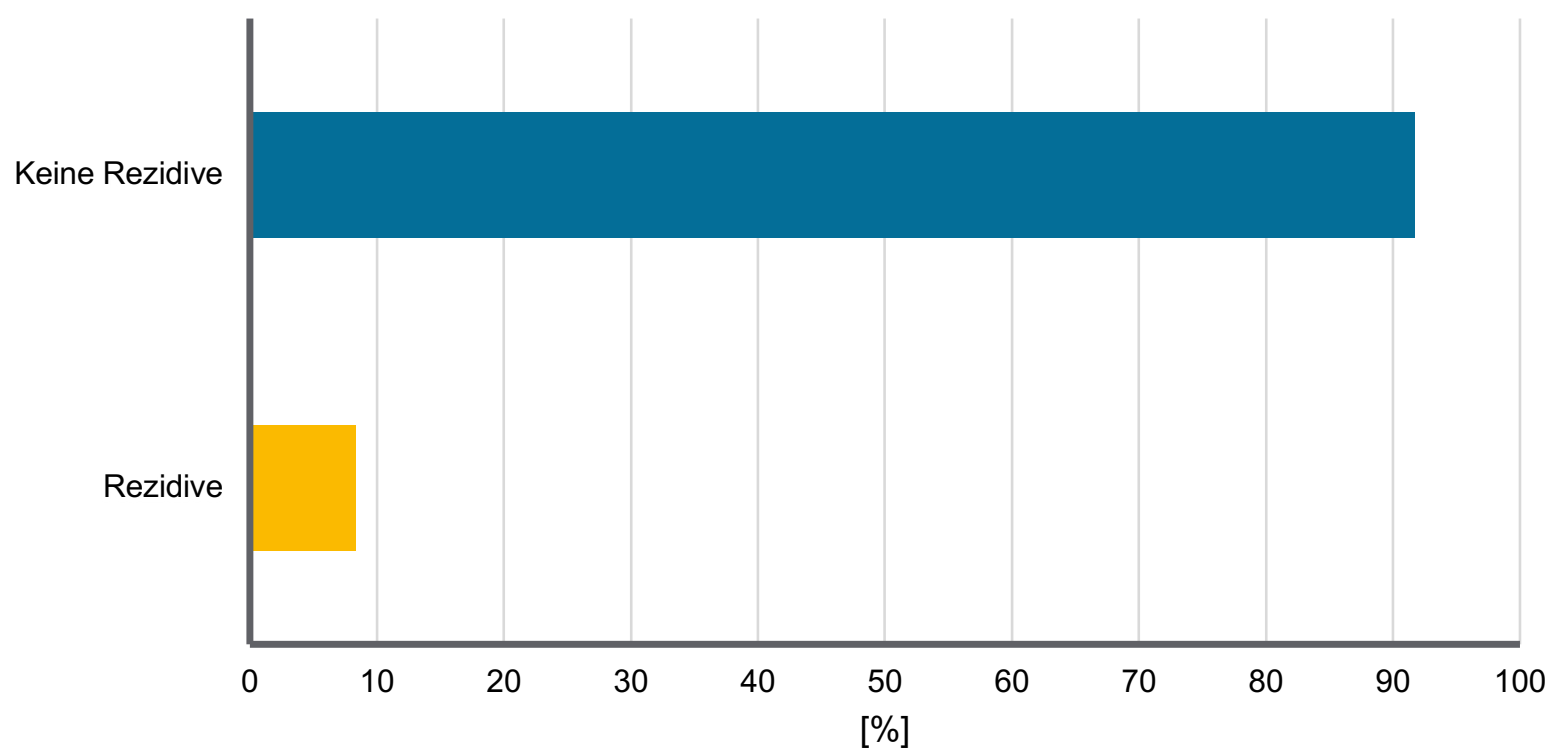

Abb. 6: Rezidiventwicklung 


\subsubsection{Rezidiventwicklung bezogen auf das Patientenalter}

Folgendes Diagramm zeigt die Verteilung der Rezidive über die Altersgruppen.

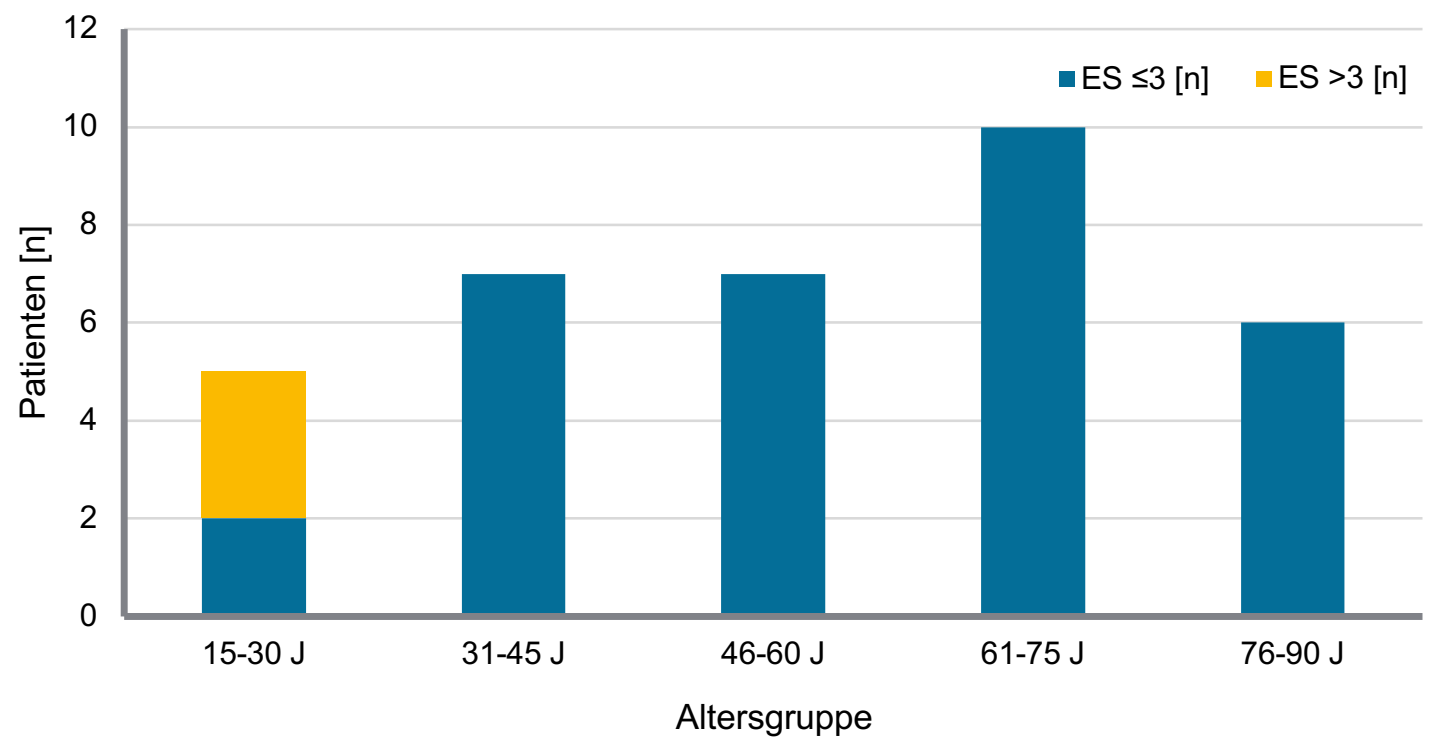

Abb. 11: Rezidiventwicklung nach Altersgruppe

Es zeigt sich ein altersbedingter Unterschied bzgl. der Rezidiventwicklung. Alle drei Patienten, die sechs Wochen postinterventionell einen Eckardt Score $>3$ aufweisen, stammen aus Altersgruppe 1 (15-30 Jahre).

Um eine signifikante Korrelation zwischen Alter und Rezidiventwicklung herzustellen, sind weitere Studien nötig.

\subsubsection{Rezidiventwicklung in Zusammenhang mit präinterventionellen klinischen Beschwerden}

In nachfolgendem Diagramm wird die Rezidiventwicklung der Patienten nach dem präinterventionell erhobenen Eckardt Score aufgeschlüsselt.

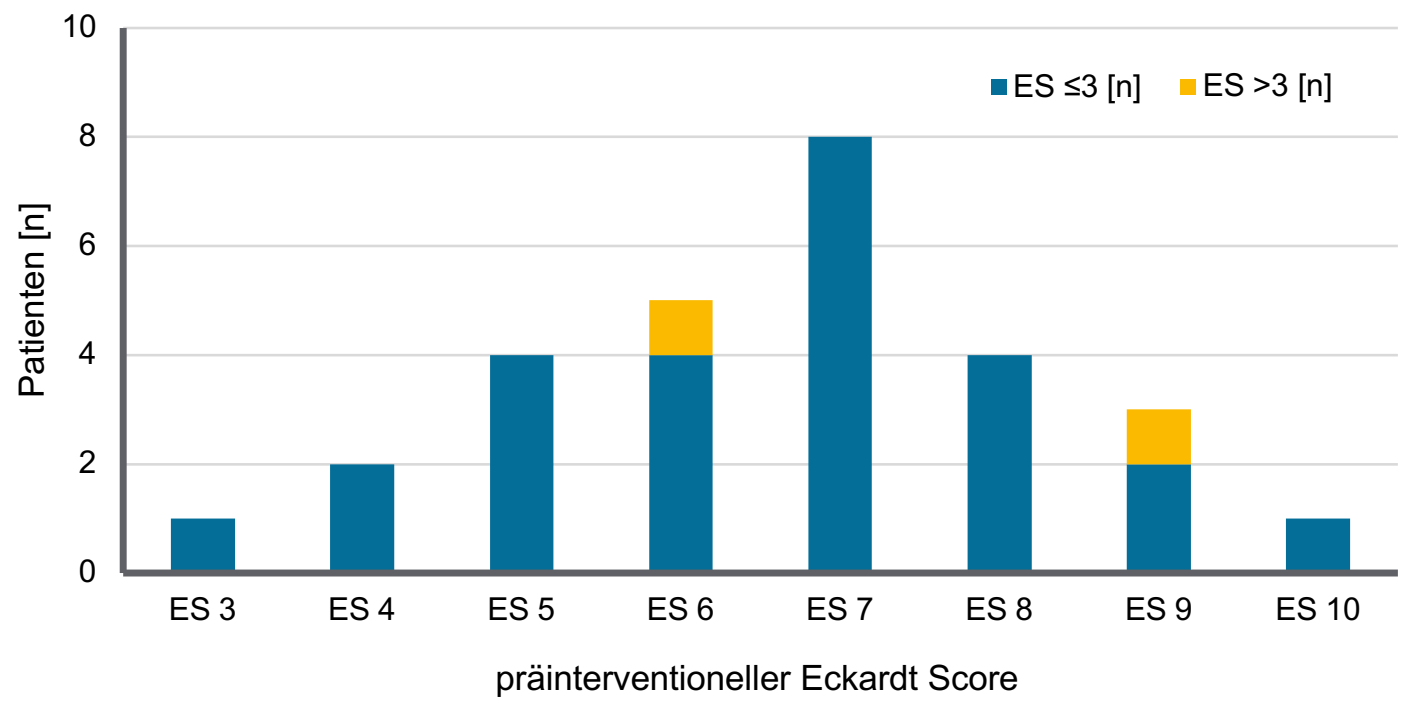

Abb. 12: Rezidiventwicklung nach präinterventionellem ES 
Es konnten 33 Patienten analysiert werden. Bei 10 Patienten (23,3\%) fehlen entsprechende Datensätze. Rezidive $(E S>3$ ) wurden jeweils bei Patienten mit einem präinterventionellen ES von 6 und 9 beobachtet. Bei einer Patientin mit Rezidiv ist kein präinterventioneller ES erhoben worden.

Um einen signifikanten Zusammenhang zwischen Schweregrad der Klinik vor POEM und der Rezidivrate herzustellen, bedarf es weiterer Studien.

\subsection{Langzeitoutcome der POEM am Ordensklinikum Linz}

Folgende Tabelle fasst das Outcome der einzelnen Jahre zusammen.

\begin{tabular}{lll}
\hline & & Anzahl, $n(\%)$ \\
\hline 2015 & & \\
& POEM gesamt & 8 \\
& $-\quad$ Komplikationen & $2(25)$ \\
& $-\quad$ Rezidive & $1(12,5)$ \\
\hline 2016 & & \\
& POEM gesamt & 10 \\
& $-\quad$ Komplikationen & $2(20)$ \\
& $-\quad$ Rezidive & $0(0)$ \\
& & \\
\hline 2017 & & 12 \\
& POEM gesamt & $1(8,3)$ \\
& $-\quad$ Komplikationen & $2(16,7)$ \\
& $-\quad$ Rezidive & \\
\hline 2018 & & 13 \\
& POEM gesamt & $0(0)$ \\
& $-\quad$ Komplikationen & $0(0)$ \\
& $-\quad$ Rezidive &
\end{tabular}

Tab.7: Komplikationen, neuaufgetretene Refluxkrankheiten und Rezidive pro Jahr

In nachfolgend angeführtem Diagramm werden Anzahl der durchgeführten Eingriffe, Komplikationen und Rezidive pro Jahr graphisch dargestellt. 


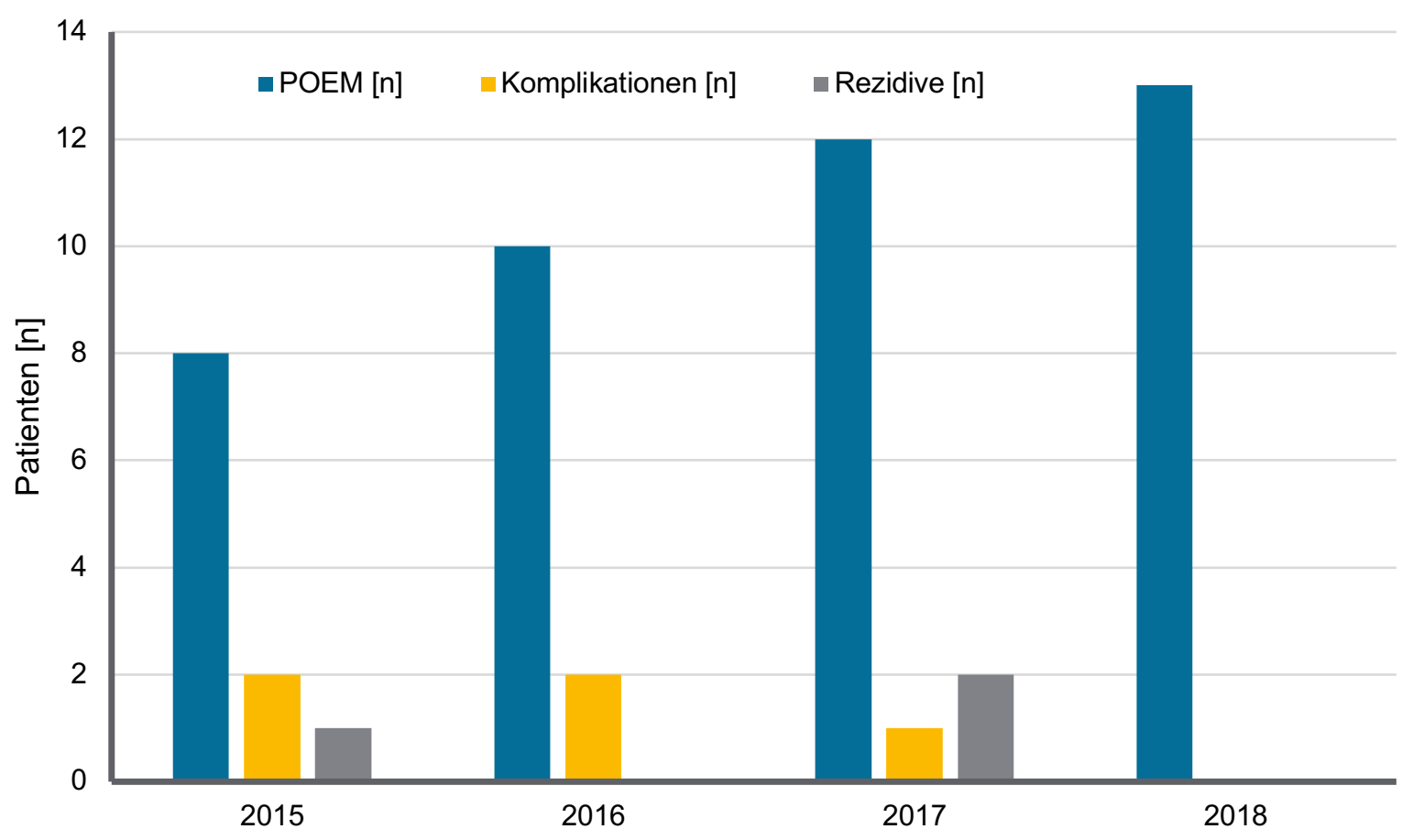

Abb. 13: Langzeitoutcome POEM am Ordensklinikum Linz

Im zeitlichen Verlauf nimmt der Anteil der Komplikationen deutlich ab. Die Komplikationsraten sind von $25 \%$ und $20 \%$ auf $8,3 \%$ und letztendlich $0 \%$ gesunken. Es zeigt sich ein Rezidiv im Jahr 2015 und zwei Rezidive im Jahr 2017. In den 13 durchgeführten Interventionen im Jahr 2018 wurde keine Komplikation und kein Rezidiv beobachtet. 


\section{Diskussion}

Die perorale endoskopische Myotomie konnte im Rahmen unserer Analyse mit einer initialen Erfolgsrate von 91,7\% ( $n=33)$ durchgeführt werden (Therapieerfolg definiert als Eckardt Score $\leq 3$ sechs Wochen nach POEM). Es konnte eine signifikante Reduktion des Eckardt Scores erreicht werden (ES 7,0 $\pm 0,3$ vs. ES 1,0 $\pm 0,2 ; p<0,0001$ ). Es wurden 3 Rezidive beobachtet (8,3\%). Inoue et al. berichten in einer prospektiven Studie mit 500 Patienten von einer Erfolgsrate von $>90 \%$ und einer Reduktion des ES im Mittel von 6,0 auf 1,0 . In einer anderen rezenten randomisierten kontrollierten Studie wurden initiale Erfolgsraten von $92 \%$ beschrieben. ${ }^{40,41}$

Die Komplikationsrate unserer Studie liegt bei 11,6\%. Die entsprechenden Raten rezenter Studien variieren von $0-30 \%$, da kein Konsens besteht, was als Komplikation gewertet werden soll. Am ehesten vergleichbar ist bereits erwähnte, von Inoue et al. durchgeführte prospektive Studie mit 500 Patienten, die eine Komplikationsrate von 3,2\% erzielte. Eine japanische multizentrische retrospektive Analyse von 1300 mit POEM behandelten Patienten beobachtete eine Rate von 3,7\%. ${ }^{6,40,42}$

Die beobachteten Komplikationen steigen in unserer Analyse mit zunehmendem Alter an (46-60 Jahre: $10 \%$ vs. $61-75$ Jahre: $15,4 \%$ vs. $76-90$ Jahre: $28,6 \%$ ). In jüngeren Altersgruppen ( $<45$ Jahre) traten keine Komplikationen auf. Dieser Zusammenhang wird auf die altersbedingte erhöhte Vulnerabilität des Gewebes und die allgemein reduzierte Regenerationsfähigkeit zurückgeführt.

Die Diskrepanz der Komplikationsrate zur aktuellen Studienlage lässt sich am ehesten durch die geringe Fallzahl bzw. fehlende Datensätze und eine der Komplexität der Methode geschuldeten Lernkurve begründen. Letzterer Verdacht erhärtet sich bei Betrachtung der Komplikationsraten der einzelnen Jahre. Diese nehmen sukzessive von $25 \%$ und $20 \%$ in den ersten beiden Jahren auf 8,3\% im Jahr 2017 sowie 0\% im Jahr 2018 ab. Die über die Zeit sinkenden Raten könnten als Ausdruck einer Lernkurve gewertet werden.

In einer prospektiven Studie mit 40 mittels POEM behandelten Patienten wurden Dauer der Intervention und Inzidenz von Mukosaperforationen untersucht, um eine Lernkurve zu definieren. Das Meistern der Technik geht bewiesenermaßen mit einer Reduktion der beiden Parameter einher, welche bei erfahrenen Endoskopisten nach ca. 20 Eingriffen konstant bleiben. ${ }^{43}$

In einer ähnlich aufgebauten prospektiven Studien mit 93 Patienten war das Plateau der Eingriffsdauer und damit das Meistern der Intervention nach 60 Eingriffen erreicht. ${ }^{44}$

Eine postinterventionelle Refluxkrankheit konnte in 37,5\% der Fälle festgestellt werden, definiert als endoskopische Diagnose einer erosiven Refluxkrankheit oder dem Vorliegen einer typischer Klinik.

Die Studienlage zur Entwicklung einer Refluxkrankheit nach POEM variiert stark. In einem aktuellen systematischen Review wurden 1542 POEMs analysiert und eine Refluxösophagitis in 35,3\% der Fälle festgestellt. Die bereits erwähnte multizentrische Studie aus Japan mit 1300 Interventionen beobachtete erosive Refluxösophagitiden in 63\% der Fälle und typische Refluxsymptomatik in 15\%.6,45 
Zusammenfassend lässt sich feststellen, dass unsere Ergebnisse hinsichtlich Therapieansprechen (Reduktion des Eckardt-Score) und Refluxentwicklung den Zahlen großer randomisierter Studien entsprechen. Dies kann als Qualitätsmerkmal erachtet werden. Die Komplikationsrate liegt im internationalen Durchschnitt, mit Verweis auf eine mögliche Lernkurve bei komplexer Intervention.

\subsection{Vor- und Nachteile der POEM gegenüber anderen Methoden}

Eine randomisierte kontrollierte Studie von Ponds et. al. aus dem Jahr 2019 vergleicht POEM und pneumatische Ballondilatation (PBD) hinsichtlich Therapieerfolg (definiert als ES $\leq 3$ und dem Ausbleiben von schwerwiegenden Komplikationen bzw. der Notwendigkeit einer erneuten Therapie im Verlauf des 2-jährigen Follow-Ups). Von 126 ausgewerteten Patienten erhielten 63 eine POEM und 63 eine PBD. Der Therapieerfolg der POEM Gruppe betrug nach zwei Jahren 92\%, verglichen mit 54\% der PBD Gruppe. Die PBD Gruppe verzeichnete zwei schwerwiegende Komplikationen, unter anderem eine Ösophagusperforation. In der POEM Gruppe wurden keine Komplikationen verzeichnet. Die Studie zeigt einen signifikanten Vorteil der POEM gegenüber der PBD in Hinblick auf Sicherheit und Effektivität. ${ }^{41}$

Schlottmann et. al. veröffentlichten 2018 einen Review Artikel, in dem das Outcome der POEM mit jenem der LHM verglichen wird. Nach 12 Monaten zeigte sich eine Besserung der Dysphagie-Symptomatik bei 93,5\% der mittels POEM behandelten Patienten, während es bei $91,0 \%$ der mittels LHM behandelten Patienten zu einer Besserung der Dysphagie Symptome kam. Nach 24 Monaten zeigten 92,7\% der POEM Gruppe versus 90,0\% der LHM Gruppe eine Besserung der Klinik. Das Ergebnis lässt darauf schließen, dass auf kurze Sicht die POEM eine etwas effektivere Methode darstellt. ${ }^{46}$

Eine Metaanalyse von Repici et. al. aus dem Jahr 2018 vergleicht die LHM und POEM hinsichtlich des Auftretens einer postoperativen Refluxsymptomatik. Von 1542 POEM Patienten präsentierten 19,0\% eine Refluxsymptomatik. Bei den 2581 LHM Patienten fiel die Refluxrate mit $8,8 \%$ deutlich niedriger aus. Die Rate von postoperativen Refluxösophagitiden war nach POEM im Vergleich zur LHM mit 29,4\% versus 7,6\% ebenfalls deutlich erhöht. ${ }^{45}$

Ein möglicher Vorteil der LHM mit Fundoplicatio gegenüber der POEM ist eine niedrigere Wahrscheinlichkeit für postoperative Refluxerkrankungen.

Um den Stellenwert der POEM zu evaluieren und LHM mit POEM zu vergleichen, werden in Zukunft Langzeit-Daten und randomisierte kontrollierte Studien notwendig sein. 


\section{Ausblick und Verbesserungsansätze}

Die Länge der Myotomie, im speziellen der gastrale Anteil derselben, ist ausschlaggebend für sowohl Therapieansprechen und Auftreten einer Refluxösophagitis. Wird die Myotomie zu kurz gewählt, steigt die Wahrscheinlichkeit für ein Rezidiv. Bei zu großzügig gewählter Myotomielänge steigt das Risiko für eine gastroösophageale Refluxkrankheit durch eine verminderte Verschlussfähigkeit des UÖS. ${ }^{47}$

Die neue Katheter-basierte EndoFlip-Methode (=endoluminal functional imaging probe) ermöglicht eine Echtzeit-Evaluierung des gastroösophagealen Übergangs während der POEM. Erhoben werden Durchmesser, Querschnittsfläche und Distensibilität im Bereich des gastroösophagealen Übergangs. In einer retrospektiven Studie von Ngamruengphong et al. wurden die Ergebnisse von 63 Patienten, welche mittels POEM und intraoperativem Endoflip behandelt wurden, ausgewertet. Bei 50 Patienten konnte ein Therapieerfolg verzeichnet werden. Die intraoperativ erhobene Querschnittsfläche des gastroösophagealen Übergangs war signifikant höher bei Therapieerfolg (Median 89.0 (IR 78.5-106.7) versus 72.4 (IR 48.8-80.0) $\mathrm{mm}^{2}$ [ $\left.p=0.01\right]$ ). Patienten mit postoperativem Reflux zeigten ebenso eine signifikant erhöhte Querschnittsfläche des gastroösophagealen Übergangs im Vergleich zu symptomfreien Patienten auf (Median 99.5 (IR 91.2-103.7) versus 79.3 (IR 57.1-94.2) $\mathrm{mm}^{2}[p=0.02]$ ). Die EndoFlip-Methode könnte zukünftig als nützliches Tool zur Prädiktion von Therapieerfolg und Refluxinduktion verwendet werden. ${ }^{47}$

Eine weitere endoskopische Methode um Länge (v.a. im Bereich des Magens) und Richtung der Myotomie besser zu kontrollieren ist die sog. „double scope“- Methode. Dabei wird ein zweites Endoskop durch den Ösophagus in den Magen eingebracht. Dies dient der Observierung der Kardia-Region in Inversion. Erreicht die Myotomie den gastroösophagealen Übergang, wird das Licht des myotomierenden Endoskops im observierenden Endoskop im Magenlumen sichtbar. Indem der Operateur sich am Abstand des durch die Mukosa einfallenden Lichts zum Zweitendoskop orientiert, kann die Länge der gastralen Myotomie präziser kontrolliert werden. ${ }^{48}$

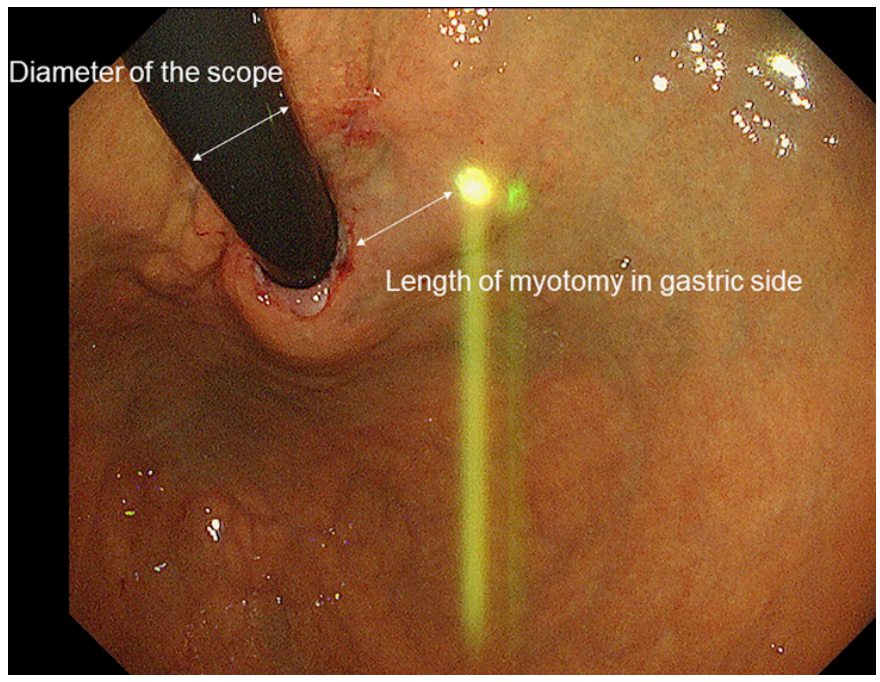

Abb. 13: Double-scope-POEM (Quelle: Inoue et al., Esophagus, Sept.19) 
Die Auswertung der Datensätze war durch das Fehlen einiger Parameter erschwert. So fehlen z.B. Daten zum Eckardt Score der Patienten. Eine Erhebung des ES vor POEM sowie im postinterventionellen Verlauf ist essentiell für das Follow-Up des Patienten und das Qualitätsmanagement der Methode.

Ebenfalls fehlen Datensätze bzgl. der Frage nach einer postinterventionellen Refluxkrankheit. Im Sinne der Patienten und zukünftiger Studien sollte im Rahmen der Kontroll-Gastroskopie eine standardisierte klinische Evaluierung einer eventuell neu aufgetretenen Refluxsymptomatik erfolgen.

Das Outcome wird bisher lediglich klinisch über den Eckardt Score evaluiert. Um objektive Aussagen treffen zu können wäre eine standardisierte HR-Manometrie nach POEM eine Möglichkeit. 


\section{Abbildungsverzeichnis}

Abb. 1: Achalasie Typ 1 (Quelle: Uniklinik Freiburg)

Abb. 2: Achalasie Typ 2 (Quelle: Ordensklinikum Linz)

Abb. 3: Achalasie Typ 3 (Quelle: Ordensklinikum Linz)

Abb. 4: Durchschnittlicher Eckardt Score vor und nach POEM

Abb. 5: Verteilung der prä- und postinterventionellen ES

Abb. 6: Refluxentwicklung nach POEM

Abb. 7: Refluxentwicklung nach Altersgruppe

Abb. 8: Verteilung des IRP im Zusammenhang mit Refluxkrankheit nach POEM

Abb. 9: Komplikationsraten nach Altersgruppe

Abb. 10: Rezidiventwicklung

Abb. 11: Rezidiventwicklung nach Altersgruppe

Abb. 12: Rezidiventwicklung nach präinterventionellem ES

Abb. 13: Langzeitoutcome POEM am Ordensklinikum Linz

Abb. 14: Double-scope POEM (Quelle: Inoue et al. ${ }^{49}$ ) 


\section{Tabellenverzeichnis}

Tab. 1: Eckardt Score (Quelle: Eigene Darstellung)

Tab. 2: Patientencharakteristika

Tab. 3: Altersgruppen

Tab. 4: Los Angeles Klassifikation der Refluxösophagitis (Quelle: Rath et al. ${ }^{39}$ )

Tab. 5: Komplikationen

Tab. 6: Komplikationen nach Altersgruppe

Tab. 7: Komplikationen, neuaufgetretene Refluxkrankheiten und Rezidive pro Jahr 


\section{Abkürzungsverzeichnis}

$\begin{array}{ll}\text { AG } & \text { Altersgruppe } \\ \text { DCI } & \text { Distal Contractile Interval } \\ \text { EBD } & \text { Endoskopische Ballondilatation } \\ \text { ERD } & \text { Erosive Refluxkrankheit (erosive reflux disease) } \\ \text { GERD } & \text { Gastroösophageale Refluxkrankheit (gastroesophageal reflux } \\ & \text { disease) } \\ \text { HRM } & \text { High-Resolution Manometrie } \\ \text { IRP } & \text { Integrated Relaxation Pressure } \\ \text { LHM } & \text { Laparoskopische Heller Myotomie } \\ \text { ÖGD } & \text { Ösophagogastroduodenoskopie } \\ \text { PBD } & \text { Pneumatische Ballondilatation } \\ \text { POEM } & \text { Perorale endoskopische Myotomie } \\ \text { PPI } & \text { Protonenpumpeninhibitor } \\ \text { UÖS } & \text { Unterer Ösophagussphinkter }\end{array}$




\section{Literaturverzeichnis}

1. von Rahden BHA, Filser J, Reimer S, Inoue H, Germer CT. Perorale endoskopische Myotomie zur Therapie der Achalasie: Literaturüberblick und eigene initiale Erfahrung. Chirurg. 2014;85(5):420-432. doi:10.1007/s00104-013-2639-0

2. Li Q-L, Chen W-F, Zhou P-H, et al. Peroral endoscopic myotomy for the treatment of achalasia: a clinical comparative study of endoscopic full-thickness and circular muscle myotomy. J Am Coll Surg. 2013;217(3):442-451. doi:10.1016/j.jamcollsurg.2013.04.033

3. Vaezi M, Krill J, Naik R. Clinical management of achalasia: current state of the art. Clin Exp Gastroenterol. 2016;9:71-82. doi:10.2147/CEG.S84019

4. Inoue $\mathrm{H}$, Minami $\mathrm{H}$, Kobayashi $\mathrm{Y}$, et al. Peroral endoscopic myotomy (POEM) for esophageal achalasia. Endoscopy. 2010;42(04):265-271. doi:10.1055/s-0029-1244080

5. von Renteln D, Inoue $\mathrm{H}$, Minami $\mathrm{H}$, et al. Peroral Endoscopic Myotomy for the Treatment of Achalasia: A Prospective Single Center Study. Am J Gastroenterol. 2012;107(3):411417. doi:10.1038/ajg.2011.388

6. Shiwaku $\mathrm{H}$, Inoue $\mathrm{H}$, Onimaru $\mathrm{M}$, et al. Multicenter collaborative retrospective evaluation of peroral endoscopic myotomy for esophageal achalasia: analysis of data from more than 1300 patients at eight facilities in Japan. Surg Endosc. June 2019. doi:10.1007/s00464019-06833-8

7. $\mathrm{Ng} \mathrm{KY}$, Li KF, Lok KH, et al. Ten-year review of epidemiology, clinical features, and treatment outcome of achalasia in a regional hospital in Hong Kong. Hong Kong Med $\mathrm{J}=$ Xianggang yi xue za zhi. 2010;16(5):362-366.

http://www.ncbi.nlm.nih.gov/pubmed/20890000. Accessed October 6, 2019.

8. Boeckxstaens GE, Zaninotto G, Richter JE. Achalasia. Lancet. 2014;383(9911):83-93. doi:10.1016/S0140-6736(13)60651-0

9. Howard PJ, Maher L, Pryde A, Cameron EW, Heading RC. Five year prospective study of the incidence, clinical features, and diagnosis of achalasia in Edinburgh. Gut. 1992;33(8):1011-1015. http://www.ncbi.nIm.nih.gov/pubmed/1398223. Accessed July 5, 2017.

10. Ho KY, Tay HH, Kang JY. A prospective study of the clinical features, manometric findings, incidence and prevalence of achalasia in Singapore. J Gastroenterol Hepatol. 1999;14(8):791-795. http://www.ncbi.nlm.nih.gov/pubmed/10482430. Accessed July 5, 2017.

11. Furuzawa-Carballeda J, Aguilar-León D, Gamboa-Domínguez A, et al. Achalasia--An Autoimmune Inflammatory Disease: A Cross-Sectional Study. J Immunol Res. 2015;2015:729217. doi:10.1155/2015/729217

12. Al-Jafar H, Laffan M, Al-Sabah S, Elmorsi M, Habeeb M, Alnajar F. Severe recurrent achalasia cardia responding to treatment of severe autoimmune acquired haemophilia. Case Rep Gastroenterol. 2012;6(3):618-623. doi:10.1159/000343435

13. Verne GN, Hahn AB, Pineau BC, Hoffman BJ, Wojciechowski BW, Wu WC. Association of HLA-DR and -DQ alleles with idiopathic achalasia. Gastroenterology. 1999;117(1):2631. http://www.ncbi.nlm.nih.gov/pubmed/10381906. Accessed June 13, 2017.

14. Kahrilas PJ, Kishk SM, Helm JF, Dodds WJ, Harig JM, Hogan WJ. Comparison of pseudoachalasia and achalasia. Am J Med. 1987;82(3):439-446.

http://www.ncbi.nlm.nih.gov/pubmed/3548347. Accessed June 14, 2017.

15. Dufresne CR, Jeyasingham K, Baker RR. Achalasia of the cardia associated with pulmonary sarcoidosis. Surgery. 1983;94(1):32-35.

http://www.ncbi.nlm.nih.gov/pubmed/6857509. Accessed June 14, 2017.

16. Costigan DJ, Clouse RE. Achalasia-like esophagus from amyloidosis. Successful treatment with pneumatic bag dilatation. Dig Dis Sci. 1983;28(8):763-765. http://www.ncbi.nlm.nih.gov/pubmed/6872809. Accessed June 14, 2017.

17. Foster PN, Stewart M, Lowe JS, Atkinson M. Achalasia like disorder of the oesophagus in von Recklinghausen's neurofibromatosis. Gut. 1987;28(11):1522-1526.

http://www.ncbi.nlm.nih.gov/pubmed/3123332. Accessed June 14, 2017.

18. Cuthbert JA, Gallagher ND, Turtle JR. Colonic and oesophageal disturbance in a patient with multiple endocrine neoplasia, type 2b. Aust N Z J Med. 1978;8(5):518-520. http://www.ncbi.nlm.nih.gov/pubmed/33647. Accessed June 14, 2017.

19. Campos CT, Ellis FH, LoCicero J. Pseudoachalasia: a report of two cases with comments on possible causes and diagnosis. Dis esophagus Off J Int Soc Dis Esophagus. 
1997;10(3):220-224. http://www.ncbi.nlm.nih.gov/pubmed/9280084. Accessed June 14, 2017.

20. Schuffler MD. Chronic intestinal pseudo-obstruction syndromes. Med Clin North Am. 1981;65(6):1331-1358. http://www.ncbi.nlm.nih.gov/pubmed/6799718. Accessed June 14, 2017.

21. Roberts DH, Gilmore IT. Achalasia in Anderson-Fabry's disease. J R Soc Med. 1984;77(5):430-431. http://www.ncbi.nlm.nih.gov/pubmed/6427465. Accessed June 14, 2017.

22. Holloway RH, Dodds WJ, Helm JF, Hogan WJ, Dent J, Arndorfer RC. Integrity of cholinergic innervation to the lower esophageal sphincter in achalasia. Gastroenterology. 1986;90(4):924-929. http://www.ncbi.nlm.nih.gov/pubmed/3949120. Accessed June 13, 2017.

23. Sodikoff JB, Lo AA, Shetuni BB, Kahrilas PJ, Yang G-Y, Pandolfino JE. Histopathologic patterns among achalasia subtypes. Neurogastroenterol Motil. 2016;28(1):139-145. doi:10.1111/nmo.12711

24. Hirano I, Tatum RP, Shi G, Sang Q, JoehI RJ, Kahrilas PJ. Manometric heterogeneity in patients with idiopathic achalasia. Gastroenterology. 2001;120(4):789-798. http://www.ncbi.nlm.nih.gov/pubmed/11231931. Accessed June 14, 2017.

25. Spechler SJ, Talley NJ, Robson KM. Achalasia: Pathogenesis, clinical manifestations, and diagnosis. http://www.uptodate.com/contents/achalasia-pathogenesis-clinicalmanifestations-and-diagnosis. Accessed June 14, 2017.

26. Uniklinik Freiburg. Typ 1 Achalasie. https://www.uniklinikfreiburg.de/medizin2/interdisziplinaere-endoskopie/spezialsprechstunde-therapeutischeendoskopie/schluckstoerungen.html. Accessed July 6, 2017.

27. Public Library of Science. H, Isomoto H, Miuma S, et al. New Endoscopic Indicator of Esophageal Achalasia: "Pinstripe Pattern." Vol 10. Public Library of Science https://doaj.org/article/d8263327f1b64d07a47ebcc5073048d2. Accessed March 7, 2017.

28. Wen ZH, Gardener E, Wang YP. Nitrates for achalasia. Wen Z, ed. Cochrane database Syst Rev. 2004;(1):CD002299. doi:10.1002/14651858.CD002299.pub2

29. Stefanidis D, Richardson W, Farrell T. Guidelines for the Surgical Treatment of Esophageal Achalasia - A SAGES Guideline.

https://www.sages.org/publications/guidelines/guidelines-for-the-surgical-treatment-ofesophageal-achalasia/. Accessed June 15, 2017.

30. Pasricha PJ, Ravich WJ, Hendrix TR, Sostre S, Jones B, Kalloo AN. Treatment of achalasia with intrasphincteric injection of botulinum toxin. A pilot trial. Ann Intern Med. 1994;121(8):590-591. http://www.ncbi.nlm.nih.gov/pubmed/8085691. Accessed June 16, 2017.

31. Leyden JE, Moss AC, MacMathuna P. Endoscopic pneumatic dilation versus botulinum toxin injection in the management of primary achalasia. In: Leyden JE, ed. Cochrane Database of Systematic Reviews. Chichester, UK: John Wiley \& Sons, Ltd; 2014. doi:10.1002/14651858.CD005046.pub3

32. Borhan-Manesh F, Kaviani MJ, Taghavi AR. The efficacy of balloon dilation in achalasia is the result of stretching of the lower esophageal sphincter, not muscular disruption. Dis esophagus Off J Int Soc Dis Esophagus. 2016;29(3):262-266. doi:10.1111/dote.12314

33. Boeckxstaens GE, Annese V, des Varannes SB, et al. Pneumatic dilation versus laparoscopic Heller's myotomy for idiopathic achalasia. N Engl J Med. 2011;364(19):1807-1816. doi:10.1056/NEJMoa1010502

34. Lopushinsky SR, Urbach DR. Pneumatic dilatation and surgical myotomy for achalasia. JAMA. 2006;296(18):2227-2233. doi:10.1001/jama.296.18.2227

35. Elliott TR, Wu PI, Fuentealba S, Szczesniak M, de Carle DJ, Cook IJ. Long-term outcome following pneumatic dilatation as initial therapy for idiopathic achalasia: an 18-year singlecentre experience. Aliment Pharmacol Ther. 2013;37(12):1210-1219.

doi:10.1111/apt.12331

36. Abir F, Modlin I, Kidd M, Bell R. Surgical treatment of achalasia: current status and controversies. Dig Surg. 2004;21(3):165-176. doi:10.1159/000079341

37. Campos GM, Vittinghoff E, Rabl C, et al. Endoscopic and surgical treatments for achalasia: a systematic review and meta-analysis. Ann Surg. 2009;249(1):45-57. doi:10.1097/SLA.0b013e31818e43ab

38. Yang D, Wagh MS. Peroral endoscopic myotomy for the treatment of achalasia: an analysis. Diagn Ther Endosc. 2013;2013:389596. doi:10.1155/2013/389596 
39. Rath HC, Timmer A, Kunkel C, et al. Comparison of interobserver agreement for different scoring systems for reflux esophagitis: Impact of level of experience. Gastrointest Endosc. 2004;60(1):44-49. doi:10.1016/s0016-5107(04)01289-1

40. Inoue $\mathrm{H}$, Sato $\mathrm{H}$, Ikeda $\mathrm{H}$, et al. Per-Oral Endoscopic Myotomy: A Series of 500 Patients. J Am Coll Surg. 2015;221(2):256-264. doi:10.1016/j.jamcollsurg.2015.03.057

41. Ponds FA, Fockens P, Lei A, et al. Effect of Peroral Endoscopic Myotomy vs Pneumatic Dilation on Symptom Severity and Treatment Outcomes Among Treatment-Naive Patients With Achalasia: A Randomized Clinical Trial. JAMA. 2019;322(2):134-144. doi:10.1001/jama.2019.8859

42. Crespin OM, Liu LWC, Parmar A, et al. Safety and efficacy of POEM for treatment of achalasia: a systematic review of the literature. Surg Endosc. 2017;31(5):2187-2201. doi:10.1007/s00464-016-5217-y

43. Kurian AA, Dunst CM, Sharata A, Bhayani NH, Reavis KM, Swanström LL. Peroral endoscopic esophageal myotomy: defining the learning curve. Gastrointest Endosc. 2013;77(5):719-725. doi:10.1016/j.gie.2012.12.006

44. Patel KS, Calixte R, Modayil RJ, Friedel D, Brathwaite CE, Stavropoulos SN. The light at the end of the tunnel: a single-operator learning curve analysis for per oral endoscopic myotomy. Gastrointest Endosc. 2015;81(5):1181-1187. doi:10.1016/j.gie.2014.10.002

45. Repici A, Fuccio L, Maselli R, et al. GERD after per-oral endoscopic myotomy as compared with Heller's myotomy with fundoplication: a systematic review with metaanalysis. Gastrointest Endosc. 2018;87(4):934-943.e18. doi:10.1016/j.gie.2017.10.022

46. Schlottmann F, Luckett DJ, Fine J, Shaheen NJ, Patti MG. Laparoscopic Heller Myotomy Versus Peroral Endoscopic Myotomy (POEM) for Achalasia. Ann Surg. 2018;267(3):451460. doi:10.1097/SLA.0000000000002311

47. Ngamruengphong S, von Rahden BHA, Filser J, et al. Intraoperative measurement of esophagogastric junction cross-sectional area by impedance planimetry correlates with clinical outcomes of peroral endoscopic myotomy for achalasia: a multicenter study. Surg Endosc. 2016;30(7):2886-2894. doi:10.1007/s00464-015-4574-2

48. Grimes KL, Inoue H, Onimaru M, et al. Double-scope per oral endoscopic myotomy (POEM): a prospective randomized controlled trial. Surg Endosc. 2016;30(4):1344-1351. doi:10.1007/s00464-015-4396-2

49. Inoue $\mathrm{H}$, Shiwaku H, Kobayashi $\mathrm{Y}$, et al. Statement for gastroesophageal reflux disease after peroral endoscopic myotomy from an international multicenter experience. Esophagus. September 2019. doi:10.1007/s10388-019-00689-6 